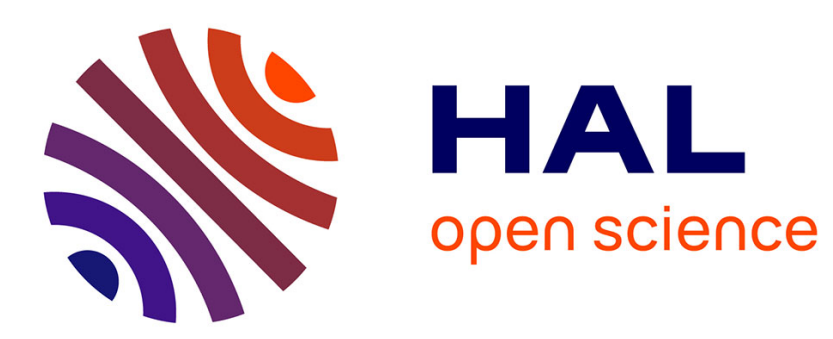

\title{
Internal controllability of systems of semilinear coupled one-dimensional wave equations with one control
}

Christophe Zhang

\section{To cite this version:}

Christophe Zhang. Internal controllability of systems of semilinear coupled one-dimensional wave equations with one control. SIAM Journal on Control and Optimization, 2018, 56 (4), pp.3092 - 3127. $10.1137 / 17 \mathrm{~m} 1128885$. hal-01517461v3

\section{HAL Id: hal-01517461 \\ https://hal.science/hal-01517461v3}

Submitted on 25 Oct 2018

HAL is a multi-disciplinary open access archive for the deposit and dissemination of scientific research documents, whether they are published or not. The documents may come from teaching and research institutions in France or abroad, or from public or private research centers.
L'archive ouverte pluridisciplinaire HAL, est destinée au dépôt et à la diffusion de documents scientifiques de niveau recherche, publiés ou non, émanant des établissements d'enseignement et de recherche français ou étrangers, des laboratoires publics ou privés. 


\title{
INTERNAL CONTROLLABILITY OF SYSTEMS OF SEMILINEAR COUPLED ONE-DIMENSIONAL WAVE EQUATIONS WITH ONE CONTROL*
}

\author{
CHRISTOPHE ZHANG $^{\dagger}$
}

\begin{abstract}
We study systems of two coupled wave equations in one space dimension, with one control, spatially supported on an arbitrarily small interval. We obtain the controllability of such systems under certain conditions on the coupling. To do this we apply the "fictitious control method" in two cases: general systems with a controllable linearized system, and a particular case where the linearized system is not controllable, namely, a cubic coupling. In the latter case, our proof requires finding nontrivial trajectories of the control system that go from 0 to 0 and having a controllable linearized system. We build these trajectories by adapting (in one space dimension) a construction developed by Jean-Michel Coron, Sergio Guerrero, and Lionel Rosier for the study of coupled parabolic systems.
\end{abstract}

Key words. wave equations, coupled systems, exact internal controllability, fictitious control method, algebraic solvability, return method

AMS subject classifications. 35L20, 93B05, 93C10

DOI. $10.1137 / 17 \mathrm{M} 1128885$

\section{Main results and outline of proof.}

1.1. Control systems. Let $T>0$, and $0<a<b<L$. We study the following class of systems:

$$
\left\{\begin{aligned}
u_{t t}-\nu_{1}^{2} u_{x x} & =f_{1}(u, v)+h, x \in(0, L), \\
v_{t t}-\nu_{2}^{2} v_{x x} & =f_{2}(u, v), x \in(0, L), \\
u & =0 \text { on }\{0, L\}, \\
v & =0 \text { on }\{0, L\},
\end{aligned}\right.
$$

where $h:[0, T] \times[0, L] \rightarrow \mathbb{R}$ is the control, with supp $h \subset[0, T] \times[a, b]$, and $f_{1}, f_{2} \in$ $C^{\infty}\left(\mathbb{R}^{2}\right), f_{1}(0,0)=f_{2}(0,0)=0, \nu_{1}, \nu_{2} \neq 0$. In what follows we shall note, for any $\nu \neq 0$

$$
\square_{\nu}:=\partial_{t t}-\nu^{2} \partial_{x x}
$$

We will also study the following particular system:

$$
\left\{\begin{aligned}
\square_{\nu_{1}} u & =h, x \in(0, L), \\
\square_{\nu_{2}} v & =u^{3}, x \in(0, L), \\
u & =0 \text { on }\{0, L\}, \\
v & =0 \text { on }\{0, L\} .
\end{aligned}\right.
$$

These are systems of coupled semilinear wave equations, with different speeds, which we seek to control with a single control, which takes the form of a source term in the first equation with a support in $[0, L] \times[a, b]$. In both cases, we will study solutions

${ }^{*}$ Received by the editors May 5, 2017; accepted for publication (in revised form) June 22, 2018; published electronically August 23, 2018.

http://www.siam.org/journals/sicon/56-4/M112888.html

Funding: This work was partially supported by ANR project Finite4SoS (ANR-15-CE23-0007).

${ }^{\dagger}$ Laboratoire Jacques-Louis Lions, Paris, 75005, France (christophe.zhang@polytechnique.org). 
with $C^{k}((0, T] \times[0, L])$ regularity in order to establish a controllability result with two controls. Thus, the initial and final conditions $\left(\left(u_{0}, u_{1}\right),\left(v_{0}, v_{1}\right),\left(u_{0}^{f}, u_{1}^{f}\right),\left(v_{0}^{f}, v_{1}^{f}\right)\right)$ have to satisfy some compatibility conditions. For example, the conditions of order 1 and 2 read as

$$
\forall \beta \in\{0, L\},\left\{\begin{array}{l}
u_{0}(\beta)=u_{1}(\beta)=\left(u_{0}^{f}\right)(\beta)=\left(u_{1}^{f}\right)(\beta)=0, \\
u_{0}^{\prime \prime}(\beta)=u_{1}^{\prime \prime}(\beta)=\left(u_{0}^{f}\right)^{\prime \prime}(\beta)=\left(u_{1}^{f}\right)^{\prime \prime}(\beta)=0, \\
v_{0}(\beta)=v_{1}(\beta)=\left(v_{0}^{f}\right)(\beta)=\left(v_{1}^{f}\right)(\beta)=0, \\
v_{0}^{\prime \prime}(\beta)=v_{1}^{\prime \prime}(\beta)=\left(v_{0}^{f}\right)^{\prime \prime}(\beta)=\left(v_{1}^{f}\right)^{\prime \prime}(\beta)=0 .
\end{array}\right.
$$

To write the compatibility conditions of order $k \geq 3$, the idea is to first write the time derivatives of $u$ and $v$ as a function of their lower order derivatives.

There exists a multivariate polynomial $Q_{n, i}^{f_{i}}$ such that

$$
\left(\frac{d}{d t}\right)^{n}\left(f_{i}(u, v)\right)=Q_{n, i}^{f_{i}}\left(J_{t}^{n}(u, v)\right), i=1,2,
$$

where $J_{t}^{n}(u, v)$ denotes the $n$-jet of time derivatives of $u$ and $v$, that is,

$$
\left(u, v, u_{t}, v_{t}, \ldots, \partial_{t}^{n} u, \partial_{t}^{n} v\right) .
$$

Now, define by recurrence the following family of operators:

$$
\left\{\begin{array}{l}
\mathcal{D}_{1}^{(i)}=\partial_{t}, \\
\mathcal{D}_{2}^{(i)}=\partial_{x x}+f_{i}(\cdot, \cdot), \\
\mathcal{D}_{n}^{(i)}=\partial_{x x} \circ \mathcal{D}_{n-2}^{(i)}+Q_{n-2, i}^{f_{i}}\left(J_{t}^{n-2}(\cdot, \cdot)\right) \text { for } 3 \leq n \leq k .
\end{array}\right.
$$

Then, near the corners $\Gamma:=\{(0,0),(0, L),(T, 0),(T, L)\}$, using the equations of system (1.1) and keeping in mind that the control $h$ is supported away from the corners, we have

$$
\left\{\begin{array}{l}
\partial_{t}^{n} u=\mathcal{D}_{n}^{(1)}(u, v), \\
\partial_{t}^{n} v=\mathcal{D}_{n}^{(2)}(u, v) .
\end{array}\right.
$$

Now, thanks to the boundary conditions,

$$
\partial_{t}^{n} u(c)=\partial_{t}^{n} v(c)=0 \quad \forall c \in \Gamma, \forall n \leq k .
$$

Moreover, it is clear thanks to the recurrence in (1.5) that there exist multivariate polynomials $P_{n, i}^{f_{i}}$ such that

$$
\mathcal{D}_{n}^{(i)}(u, v)=P_{n, i}^{f_{i}}\left(J_{x}^{n}(u, v), J_{x}^{n-1}\left(u_{t}, v_{t}\right), J_{t}^{n}(u, v)\right) \quad \forall n \leq k, i=1,2,
$$

where $J_{x}^{n}(u, v)$ denotes the $n$-jet of space derivatives $\mathrm{f} u$ and $v$. Now, (1.6) can be written in the corners using only $u_{0}, u_{1}, u_{0}^{f}, u_{1}^{f}, v_{0}, v_{1}, v_{0}^{f}, v_{1}^{f}$, which gives the following compatibility conditions of order $k$ :

$$
\left\{\begin{array}{rl}
P_{n, i}^{f_{i}}\left(J_{x}^{n}\left(u_{0}, v_{0}\right)(0), J_{x}^{n-1}\left(u_{1}, v_{1}\right)(0),(0, \ldots, 0)\right)=0 \\
P_{n, i}^{f_{i}}\left(J_{x}^{n}\left(u_{0}, v_{0}\right)(L), J_{x}^{n-1}\left(u_{1}, v_{1}\right)(L),(0, \ldots, 0)\right)=0, \\
P_{n, i}^{f_{i}}\left(J_{x}^{n}\left(u_{0}^{f}, v_{0}^{f}\right)(0), J_{x}^{n-1}\left(u_{1}^{f}, v_{1}^{f}\right)(0),(0, \ldots, 0)\right)=0, \\
P_{n, i}^{f_{i}}\left(J_{x}^{n}\left(u_{0}^{f}, v_{0}^{f}\right)(L), J_{x}^{n-1}\left(u_{1}^{f}, v_{1}^{f}\right)(L),(0, \ldots, 0)\right)=0,
\end{array} \quad \forall n \leq k, i=1,2 .\right.
$$


The existence and uniqueness of solutions to these systems can be derived from Li's general results on quasi-linear wave equations (see [LR03] or [Li10, Chapter 5, section $5.2])$.

In this paper we prove two controllability results: a local result for system (1.1) and a global result for system (1.2).

TheOREM 1.1. Let $R>0$, and $0 \leq a<b \leq L, T>0$ such that

$$
T>2(L-b) \max \left(\frac{1}{\left|\nu_{1}\right|}, \frac{1}{\left|\nu_{2}\right|}\right), T>2 a \max \left(\frac{1}{\left|\nu_{1}\right|}, \frac{1}{\left|\nu_{2}\right|}\right) .
$$

If

$$
\frac{\partial f_{2}}{\partial u}(0,0) \neq 0
$$

then there exists $\eta>0$ such that for initial and final conditions

$$
\left(\left(u_{0}, u_{1}\right),\left(v_{0}, v_{1}\right),\left(u_{0}^{f}, u_{1}^{f}\right),\left(v_{0}^{f}, v_{1}^{f}\right)\right) \in\left(B_{C^{11}([0, L])} \times B_{C^{10}([0, L])}(0, \eta)\right)^{4},
$$

where $B_{C^{k}}(0, \eta)$ denotes the ball centered in 0 and with radius $\eta$ in the usual $C^{k}$ topology, satisfying $(1.8)$ at the order 11 , there exists $h \in C^{6}([0, T] \times[0, L])$ such that

$$
\operatorname{supp} h \subset[0, T] \times[a, b]
$$

and such that the corresponding solution $(u, v) \in C^{6}([0, T] \times[0, L])^{2}$ of $(1.1)$ with initial values $\left(\left(u_{0}, u_{1}\right),\left(v_{0}, v_{1}\right)\right)$ satisfies

$$
\begin{cases}u(T, \cdot)=u_{0}^{f}, & u_{t}(T, \cdot)=u_{1}^{f}, \\ v(T, \cdot)=v_{0}^{f}, & v_{t}(T, \cdot)=v_{1}^{f},\end{cases}
$$

and

$$
\|(u, v, h)\|_{\left(C^{6}\right)^{3}} \leq R .
$$

Condition (1.10) is necessary and sufficient for the controllability of linear systems (if the dynamics of $v$ does not depend on $u$ there is no hope to control $v$ through $u$ ). In contrast, the following theorem shows that it is not necessary in the case of nonlinear systems: system (1.2) does not satisfy (1.10), but we still obtain a controllability result. Moreover, thanks to the system's homogeneity, the result is global.

TheOREM 1.2. Let $0 \leq a<b \leq L, T>0$ satisfying (1.9). There exists a constant $C>0$ depending on $T$ such that, for any given initial and final conditions

$$
\left(\left(u_{0}, u_{1}\right),\left(v_{0}, v_{1}\right),\left(u_{0}^{f}, u_{1}^{f}\right),\left(v_{0}^{f}, v_{1}^{f}\right)\right) \in\left(C^{11}([0, L]) \times C^{10}([0, L])\right)^{4}
$$

satisfying (1.8) at the order 11 , there exists $h \in C^{6}([0, T] \times[0, L])$ such that

$$
\operatorname{supp} h \subset[0, T] \times[a, b],
$$

and such that the corresponding solution $(u, v) \in C^{6}([0, T] \times[0, L])^{2}$ of $(1.2)$ with initial values $\left(\left(u_{0}, u_{1}\right),\left(v_{0}, v_{1}\right)\right)$ satisfies

$$
\begin{cases}u(T, \cdot)=u_{0}^{f}, & u_{t}(T, \cdot)=u_{1}^{f}, \\ v(T, \cdot)=v_{0}^{f}, & v_{t}(T, \cdot)=v_{1}^{f},\end{cases}
$$


and

$$
\|h\|_{C^{6}} \leq C\left(\left\|\left(u_{0}, u_{1}, u_{0}^{f}, u_{1}^{f}\right)\right\|_{\left(C^{11} \times C^{10}\right)^{2}}+\left\|\left(v_{0}, v_{1}, v_{0}^{f}, v_{1}^{f}\right)\right\|_{\left(C^{11} \times C^{10}\right)^{2}}^{\frac{1}{3}}\right) .
$$

\subsection{Related results.}

Control of hyperbolic equations. Fundamental results for the controllability and stabilization of the linear wave equation can be found in [BLR92]. For quasi-linear wave equations, boundary controllability results for scalar systems with $C^{2}$ regularity can be found in [Li10, Chapter 5] and can be adapted to coupled systems with the same number of controls and equations, and for $C^{k}$ regularity. For the semilinear wave equation, local controllability results have been obtained using the implicit function theorem (see [Fat75] and the generalization by [Che76]). To get global boundary and internal controllability for the semilinear wave equation, under some growth constraints on the nonlinearity, Zuazua used the Hilbert uniqueness method (HUM) and introduced a suitable fixed-point method in [Zua93] and [Zua91]. These results have since then been improved successively by [CKL02] and [CKL99], where authors study the one-sided and internal controllability of a semilinear wave equation with an iterated logarithm nonlinearity. Another powerful method to prove controllability results is the Carleman estimates method. It was first used for the semilinear wave equation in [Zha00a] and [Zha00b], where a new Carleman estimate was established to prove internal observability. The estimate worked for globally Lipschitz nonlinearities, with the observer supported in a neighborhood of some portion of the boundary. More recently, Carleman estimates were used in [FYZ07] to obtain internal controllability of the semilinear wave equation in any space dimension. The control is supported in a neighborhood of a portion of the boundary (earlier works required the controller to be supported in the neighborhood of the whole boundary), and the nonlinearity is superlinear. The method of Carleman estimates was also used in [HI11] for mechanical systems of several coupled linear hyperbolic equations (a multilayer Rao-Nakra plate). This yields internal controllability results, with the same number of controls and equations, and controllers supported on an arbitrarily small neighborhood of some portion of the boundary.

Systems with fewer controls than equations. Linear case. Regarding controllability with a reduced number of controls, results for boundary and internal control of coupled linear symmetric wave systems have been proved by Alabau-Boussouira ([ABL13] and [AB13]) in any space dimension, using energy methods, with more or less strong assumptions on the coupling operators, and in particular in the case where the control domain and the coupling domain do not intersect. This was then used in [AB14] to prove the existence of insensitizing controls for a single wave equation, as this is linked to the controllability of linear cascade systems in one space dimension, with the same speed in both equations. Other methods have been used to deal with a reduced number of controllers, albeit on different types of systems: on the related question of partial observability on a sphere, on top of some results proven by Lions in [Lio88a] and [Lio88b], [KL00] shows a way to deal with a reduced number of controllers using the Fourier expansion of the solutions. They prove that for a generic choice of coupling parameters, and provided the initial conditions of the unobserved components are zero and the initial conditions of the observed components are orthogonal to a finite-dimensional space (possibly trivial, for example, in the one-dimensional (1-D) case), then partial observability holds.

Nonlinear case. The link between cascade controllability and desensitizing controls has also been explored for semilinear equations in [Teb11], where the author 
proves the controllability of cascade systems of the form

$$
\left\{\begin{aligned}
\square u+f(u) & =h+\xi, \\
\square v+f^{\prime}(u) v & =0, \\
u=0, v(t, 0) & =\frac{\partial u}{\partial n} \chi_{\Gamma_{0}} \text { on } \partial \Omega,
\end{aligned}\right.
$$

where $\Gamma_{0}$ is a portion of the boundary and where $f$ is subject to a growth constraint to have global well-posedness. To prove the controllability of such systems, the author first establishes the controllability of a linear problem, using a form of HUM combined with Carleman estimates. Then, using the Schauder fixed-point theorem, he establishes the controllability of the nonlinear problem.

In other cases, as for system (1.2), the linearized system around 0 is not controllable. A classical tool to handle this problem in finite dimension is the use of iterated Lie brackets; see, for example, [Isi95, Chapter 2], [NvdS90, Chapter 3], and [Cor07, Chapter 3]. However, this tool does not work for many partial differential equations (see, for example, [Cor07, Chapter 5]). In particular it does not work for our control system (1.2). In that case, a method to handle this situation is the return method. It consists in looking for trajectories going from 0 to 0 and such that the linearized system around them is controllable (return trajectories). This method has been introduced in [Cor92] for the stabilization of driftless control systems and in [Cor96] and in [Cor93] for the controllability of the Euler equations of incompressible fluids. It is also used in [CGR10] for parabolic systems with cubic coupling. Following this method, in [CGR10] the authors build return trajectories, using the structure of the coupling. Then, using Carleman estimates, they prove the controllability of a family of related parabolic linear systems close to the return trajectory, from which they deduce null-controllability using Kakutani's fixed-point theorem.

In yet other cases, a phenomenon of loss of derivatives can occur: this can be handled with an inversion theorem of the Nash-Moser type, with a stronger condition on the linearized system. A well-known case is the local controllability of the 1-D Schrödinger equation, which was proved in [Bea05] and [BC06] using a Nash-Moser implicit function theorem. More recently, the controllability of a system of coupled quasi-linear first order hyperbolic systems with one control was proved in [ABCO17], using the "fictitious control method" and a Nash-Moser type inversion theorem proven by Gromov, which we will explain in the following section. More precisely the result concerns systems of the form

$$
\left\{\begin{array}{l}
u_{t}+\Lambda_{1}(u, v)(u, v)+f_{1}(u, v)=h, \\
v_{t}+\Lambda_{2}(u, v)(u, v)+f_{2}(u, v)=0,
\end{array}\right.
$$

with

$$
\frac{\partial f_{2}}{\partial u}(0,0) \neq 0
$$

The work presented in this article draws from all these situations: we study semilinear systems, as in [Teb11], but of a more general form than (1.15). The idea would then be to prove a controllability result for some sort of linearized system, then use a fixed-point theorem (or an inversion theorem) to conclude. However, because of a phenomenon of loss of derivatives, we rather follow the same path as [ABCO17] to get Theorem 1.1. Using Nash-Moser type theorems to deal with the loss 
of derivatives can lead to technical developments, as in [Bea05] and [BC06]. However, in our case, the theorem of Gromov we use is more "user-friendly" and requires less work to use. Thus our method has the advantage of not being as computation heavy as Carleman estimates and allows for general control domains, whereas the use of Carleman estimates usually requires the control domain to be the neighborhood of some portion of the boundary. For Theorem 1.2, however, condition (1.10) (analogous to (1.16)) is not satisfied. This corresponds to the fact that the linearized system is not controllable, and so we build return trajectories as in [CGR10].

Finally, a remark on the control time is in order: for hyperbolic systems, the control time is usually linked to the speeds of propagation and the size of the domain, as this represents how fast the deformation produced by the control reaches every point of the domain. Now, in the case of a reduced number of controls, one can expect that the indirect action of the control should mean additional control time, or that the control time should depend not only on the geometry of the domains and the propagation speeds but on some other parameters. For example, in the results of [ABL13], the authors point out that the control time they obtain depends on all the parameters of the system, not only the geometry of the control and coupling domains. Likewise, in [AB13] and [AB14] the control times depend on observability times not only for a single equation but also for the coupling operator, and in [Teb11], the control time depends on the choice of some function used to establish Carleman estimates. On the other hand, in [ABCO17] as well as in our theorems, the control time is the same as for scalar equations. Indeed, as we will see in what follows, applying the fictitious control method does not change the control time when removing one control, and the control time depends only on the size of the support of the control. Physically speaking, we use the coupling to transmit information from one equation to another (this corresponds to conditions (1.10) and (1.16)) everywhere in the domain, so that the action of the control on the first equation can be transmitted without delay.

1.3. The fictitious control method. The fictitious control method was introduced in [Cor92] and [GBPGa05] and successfully used in [CL14], [ABCO17], and [CG17]. The idea is to first prove a controllability result with two controls (the fictitious controls), then reduce the number of controls, using some sort of fixed-point theorem, namely, Theorem 2.1.

In this article, we apply it to second order hyperbolic systems, which present the same problem of loss of derivatives as the systems in [ABCO17]. This loss of derivatives is handled by using Gromov's notion of algebraic solvability, which allows for differential operators to be inverted in a special way under some condition (infinitesimal inversion) on their derivative. This yields local results around the equilibrium, but we will also work around trajectories other than the stationary trajectory at the equilibrium, in the spirit of the return method, paying close attention to the regularities involved. Indeed, condition (1.10) from Theorem 1.1 is identical to condition (1.16) and is crucial to solve the system algebraically (see Proposition 2.2). If, as in Theorem 1.2, it is not satisfied, then, following the spirit of the return method, one can build trajectories of the system along which such a condition is verified, at least on some appropriate spatial domain.

We can thus sum up our strategy of proof in three steps:

1. Find smooth trajectories around which Theorem 2.1 can be used (when necessary).

2. Prove a local controllability result with two controls (fictitious controls) around the return trajectory, using classical boundary control results.

3. Use Theorem 2.1 to reduce the number of controls to one. 
Remark 1.1. In this method, the controllability of the linearized system is not used directly to obtain controllability of the nonlinear system using a fixed-point theorem. Rather, the corresponding condition (1.10) gives us some sort of indication that information is "well transmitted" from the first equation to the second equation so that what happens with one control in each equation can be translated into a single control in the first equation.

This article is organized as follows. In section 2, we illustrate Gromov's ideas on a linear example and then prove Theorem 1.1, which is a case where we do not need to find return trajectories. This will allow us to present how Gromov's ideas can be applied to a system of nonlinear wave equations. In section 3 we prove Theorem 1.2. In this case we need to find return trajectories, and the application of Theorem 2.1 around those trajectories will require a more detailed knowledge of the supports of the return trajectory. Finally section 4 is devoted to possible improvements and further questions on this topic.

2. First case: The linearized system is controllable. As mentioned in section 1.3, we build on the method presented in [ABCO17]. One of the main ingredients of this method is the theory of differential operators, and the notion of algebraic solvability, which we briefly present in the subsection below. The use of algebraic solvability in the study of control systems first appears in [Cor92], where it was used to prove the stabilizability of finite-dimensional systems without drift with time-varying feedbacks. It was first used in the context of partial differential equations in [CL14] for the control of the Navier-Stokes equation.

But first let us give an informal explanation of our method in the case of a linear system. First we have to rewrite the control problem using differential operators. We note $\mathscr{D}$ the operator associated with the equation of our control problem. Then, the control problem, given initial and final conditions, consists in finding $(u, v)$ with those initial and final conditions, and a control $h$ such that

$$
\mathscr{D}(u, v, h)=0 .
$$

This corresponds to an inversion problem, but with a twist: one has to find an inverse image with the right initial and final conditions. Now, using the solutions to forwardand backward-evolving Cauchy problems corresponding to the initial and final conditions, one can build functions $(u, v)$ with the right initial and final conditions. The nonlinear version of this is done at the beginning of subsection 2.2. In general, one can do this so that for some $\eta>0$,

$$
\left(h_{1}, h_{2}\right):=\mathscr{D}(u, v, 0)=0 \quad \forall t \notin[\eta, T-\eta] .
$$

Now suppose $\mathscr{D}$ is invertible. We can make the following computation, the nonlinear version of which is made in subsection 2.2 :

$$
\mathscr{D}\left((u, v, 0)+\mathscr{D}^{-1}\left(-h_{1},-h_{2}\right)\right)=\left(h_{1}, h_{2}\right)-\left(h_{1}, h_{2}\right)=0 .
$$

This seems to yield a solution to the control problem; however, we still need to check that the "corrective term" does not change the initial and final conditions. This is where Gromov's notion of algebraic solvability comes into play: the right property for $\mathscr{D}$ is not to be invertible but to be algebraically solvable. That is, the inverse can also be written as a differential operator:

$$
\mathscr{D}^{-1}\left(-h_{1},-h_{2}\right)=\sum_{r} a_{r} \partial_{r}\left(-h_{1}\right)+\sum_{r} b_{r} \partial_{r}\left(-h_{2}\right)
$$


for some functions $a_{r}, b_{r}$. With this additional property, one can see that, because $-h_{1},-h_{2}$ vanish for $t \notin[\eta, T-\eta]$,

$$
\mathscr{D}^{-1}\left(-h_{1},-h_{2}\right)=0 \quad \forall t \notin[\eta, T-\eta] .
$$

Hence, $(u, v, 0)+\mathscr{D}^{-1}\left(-h_{1},-h_{2}\right)$ still has the right initial and final conditions.

2.1. Differential relations and Gromov's theorem. In this section we sum up some basic notions regarding differential operators and Gromov's local inversion theorem for differential operators. More details can be found in [Gro86].

In what follows, $\mathcal{Q}$ is the closure of a nonempty open bounded smooth subset of $\mathbb{R}^{2}$, and $p, q, r \in \mathbb{N}^{*}$. We note $n_{r, p}:=2+p \operatorname{card}\left\{\left(\alpha_{1}, \alpha_{2}\right) \in \mathbb{N}^{2} \mid \alpha_{1}+\alpha_{2} \leq r\right\}$. Recall the definition of the $r$-jet of a function $z \in C^{r}(\mathcal{Q})^{p}$ :

$$
J^{r} z(t, x)=\left((t, x), z(t, x), \ldots, \frac{\partial^{|\alpha|} z}{\partial t^{\alpha_{1}} \partial x^{\alpha_{2}}}, \ldots, \frac{\partial^{r} z}{\partial t^{\alpha_{1}} \partial x^{\alpha_{2}}}\right) \in \mathbb{R}^{n_{r, p}} \quad \forall(t, x) \in \mathcal{Q} .
$$

Definition 2.1. A map $\mathscr{D}: C^{r}(\mathcal{Q})^{p} \rightarrow C^{0}(\mathcal{Q})^{q}$ is a $C^{\infty}$ nonlinear differential operator of order $r$ if there exists $F \in C^{\infty}\left(\mathbb{R}^{n_{r, p}}, \mathbb{R}^{q}\right)$ such that

$$
\mathscr{D}(z)=F\left(J^{r} z\right) \forall z \in C^{r}(\mathcal{Q})^{p} .
$$

This clearly implies that $\mathscr{D}$ is $C^{\infty}$ (with the usual $C^{r}, C^{0}$ topologies), and we denote by

$$
\mathscr{L}_{z}: C^{r}(\mathcal{Q})^{p} \rightarrow C^{0}(\mathcal{Q})^{q}
$$

its Fréchet differential at $z \in C^{r}(\mathcal{Q})^{p}$.

We now define some sort of manifold, over which we can invert these operators.

Definition 2.2. A subset $\mathcal{A}$ of $C^{d}(\mathcal{Q})^{p}$ is a differential relation of order $d \in \mathbb{N}$ if there exists $\mathcal{R} \subset \mathbb{R}^{n_{d, p}}$ such that

$$
\mathcal{A}=\left\{z \in C^{d}(\mathcal{Q})^{p} \mid \forall(t, x) \in \mathcal{Q}, J^{d} z(t, x) \in \mathcal{R}\right\} .
$$

It is said to be open if $\mathcal{R}$ is an open subset of $\mathbb{R}^{n_{d, p}}$. For $k \in \mathbb{N}$, we note

$$
\mathcal{A}^{k}:=\mathcal{A} \cap C^{k}(\mathcal{Q})^{p} .
$$

For classical local inversion theorems, one needs the differential at one point to be invertible. Here the requirement is somewhat stronger: we need the differential at any point to be invertible, with the extra property that the inverse of each differential is also a linear differential operator.

Definition 2.3. Let $\mathcal{A} \subset C^{d}(\mathcal{Q})^{p}$ be a differential relation of order $d$, and let $\mathscr{D}$ be a differential operator of order $r$. We say that $\mathscr{D}$ admits an infinitesimal inversion of order $s \in \mathbb{N}$ over $\mathcal{A}$ if there exists a family of linear differential operators of order $s$,

$$
z \in \mathcal{A}, \mathscr{M}_{z}: C^{s}(\mathcal{Q})^{q} \rightarrow C^{0}(\mathcal{Q})^{p},
$$

such that

1. for every $g \in C^{s}(\mathcal{Q})^{q}, z \mapsto \mathscr{M}_{z}(g)$ is a differential operator of order d (possibly nonlinear) and it is a $C^{\infty}$-differential operator in $(z, g)$,

2. (Algebraic solvability) for every $z \in \mathcal{A}^{d+r}$,

$$
\mathscr{L}_{z} \circ \mathscr{M}_{z}=I d_{C^{r+s}(\mathcal{Q})} .
$$


We can now state Gromov's inversion theorem (see [Gro86, section 2.3.2, main theorem]).

TheOrem 2.1 (Gromov). Let $\mathcal{A} \subset C^{d}(\mathcal{Q})^{p}$ be a nonempty open differential relation of order $d$, and let $\mathscr{D}$ be a differential operator of order $r$. Assume that $\mathscr{D}$ admits an infinitesimal inversion of order $s$ over $\mathcal{A}$. Let

$$
\begin{gathered}
\sigma_{0}>\max (d, 2 r+s), \\
\nu \in(0, \infty) .
\end{gathered}
$$

Then, there exists a family of sets $\mathcal{B}_{z} \subset C^{\sigma_{0}+s}(\mathcal{Q})^{q}$ and a family of operators $\mathscr{D}_{z}^{-1}$ : $\mathcal{B}_{z} \rightarrow \mathcal{A}$ where $z \in \mathcal{A}^{\sigma_{0}+r+s}$, such that the following hold:

1. (Neighborhood property) For every $z \in \mathcal{A}^{\sigma_{0}+r+s}, 0 \in \mathcal{B}_{z}$ and

$$
\mathcal{B}:=\bigcup_{z \in \mathcal{A}^{\sigma_{0}+r+s}}\{z\} \times \mathcal{B}_{z}
$$

is an open subset of $C^{\sigma_{0}+r+s}(\mathcal{Q})^{p} \times C^{\sigma_{0}+s}(\mathcal{Q})^{q}$.

2. (Inversion property)

$$
\mathscr{D}\left(\mathscr{D}_{z}^{-1}(g)\right)=\mathscr{D}(z)+g \quad \forall(z, g) \in \mathcal{B} .
$$

3. (Normalization property)

$$
\mathscr{D}_{z}^{-1}(0)=z \quad \forall z \in \mathcal{A}^{\sigma_{0}+r+s} .
$$

4. (Regularity and continuity) Let $\sigma_{0} \leq \sigma_{1} \leq \eta_{1}$; then for all $z \in \mathcal{A}^{\eta_{1}+r+s}$ and $g \in \mathcal{B}_{z}^{\sigma_{1}+s}:=\mathcal{B}_{z} \cap C^{\sigma_{1}+s}$,

$$
\mathscr{D}_{z}^{-1}(g) \in \mathcal{A}^{k} \quad \forall k<\sigma_{1} .
$$

Moreover,

$$
(z, g) \mapsto \mathscr{D}_{z}^{-1}(g) \in C^{0}\left(\mathcal{A}^{\sigma_{0}+r+s} \times \mathcal{B}_{z}^{\sigma_{1}+s}, \mathcal{A}^{k}\right) \quad \forall k<\sigma_{1} .
$$

Finally, if $\eta_{1}>\sigma_{1}$, then (2.5) and (2.6) hold for $k=\sigma_{1}$.

5. (Locality) For every $(t, x) \in \mathcal{Q}$, and for every $\left(z_{1}, g_{1}\right),\left(z_{2}, g_{2}\right) \in \mathcal{B}$, if we have

$$
\left(z_{1}, g_{1}\right)(\tilde{t}, \tilde{x})=\left(z_{2}, g_{2}\right)(\tilde{t}, \tilde{x}) \quad \forall(\tilde{t}, \tilde{x}) \in B((t, x), \nu) \cap \mathcal{Q},
$$

then

$$
\mathscr{D}_{z_{1}}^{-1}\left(g_{1}\right)(t, x)=\mathscr{D}_{z_{2}}^{-1}\left(g_{2}\right)(t, x) .
$$

Remark 2.1. The neighborhood property allows us to relate the domains of inversion for each local inversion to each other: local inverses at two "neighboring" points will be defined on domains that have "neighboring" sizes. In particular that means the domains of inversions are bound to overlap. The locality property tells us that when this happens (albeit locally), the images of the local inverses agree locally. In the linear case, this corresponds to the fact that when a function vanishes on an open set, its image by any linear differential operator also vanishes on this open set (see the beginning of the section). 
2.2. From two controls to one: Algebraic solvability. As in the linear case, we first build a trajectory $(u, v)$ with the right initial and final conditions, but with $\mathscr{D}(u, v, 0)$ potentially nonzero on some restricted domain. In terms of control theory, this amounts to solving the control problem with two controls (the fictitious controls), with restricted supports. In fact, for systems of the form

$$
\left\{\begin{aligned}
\square_{\nu_{1}} u & =f_{1}(u, v)+h_{1}, x \in[0, L], \\
\square_{\nu_{2}} v & =f_{2}(u, v)+h_{2}, x \in[0, L], \\
u & =0 \text { on }\{0, L\}, \\
v & =0 \text { on }\{0, L\},
\end{aligned}\right.
$$

where $f_{1}(0,0)=f_{2}(0,0)=0$, we have the following local controllability result, which is a consequence of boundary control results presented in [Li10, Chapter 5, sections 5.2 and 5.3].

Proposition 2.1. Let $k \geq 2,0 \leq a<b \leq L, T>0$ such that (1.9) holds. For every $0<\delta<\min (T / 2,(b-a) / 2)$ satisfying

$$
\begin{aligned}
& T-2 \delta>2(L-b+2 \delta) \max \left(\frac{1}{\left|\nu_{1}\right|}, \frac{1}{\left|\nu_{2}\right|}\right), \\
& T-2 \delta>2(a+2 \delta) \max \left(\frac{1}{\left|\nu_{1}\right|}, \frac{1}{\left|\nu_{2}\right|}\right),
\end{aligned}
$$

there exists $\eta>0$ such that, for initial and final conditions

$\left(\left(u_{0}, u_{1}\right),\left(v_{0}, v_{1}\right),\left(u_{0}^{f}, u_{1}^{f}\right),\left(v_{0}^{f}, v_{1}^{f}\right)\right) \in\left(B_{C^{k}([0, T] \times[0, L])}(0, \eta) \times B_{C^{k-1}([0, T] \times[0, L])}(0, \eta)\right)^{4}$

satisfying (1.8) at the order $k$, there exist controls $h_{1}, h_{2} \in C^{k-2}([0, T] \times[0, L])$ and constants $C_{1}, C_{2}>0$ depending on $T, \delta, k$ satisfying

$$
\begin{array}{ll}
\operatorname{supp} h_{i} \subset[\delta, T-\delta] \times[a+\delta, b-\delta], & i=1,2, \\
\left\|h_{i}\right\|_{C^{k-2}} \leq C_{1}\left\|\left(\left(u_{0}, u_{1}\right),\left(v_{0}, v_{1}\right),\left(u_{0}^{f}, u_{1}^{f}\right),\left(v_{0}^{f}, v_{1}^{f}\right)\right)\right\|_{\left(C^{k} \times C^{k-1}\right)^{4}}, & i=1,2,
\end{array}
$$

such that the corresponding solution of (2.7) with initial values $\left(\left(u_{0}, u_{1}\right),\left(v_{0}, v_{1}\right)\right)$ satisfies

$$
\begin{aligned}
& \begin{cases}u(T, \cdot)=u_{0}^{f}, & u_{t}(T, \cdot)=u_{1}^{f}, \\
v(T, \cdot)=v_{0}^{f}, & v_{t}(T, \cdot)=v_{1}^{f},\end{cases} \\
& \|(u, v)\|_{\left(C^{k}\right)^{2}} \leq C_{2}\left\|\left(\left(u_{0}, u_{1}\right),\left(v_{0}, v_{1}\right),\left(u_{0}^{f}, u_{1}^{f}\right),\left(v_{0}^{f}, v_{1}^{f}\right)\right)\right\|_{\left(C^{k} \times C^{k-1}\right)^{4}} .
\end{aligned}
$$

This result is a particular case of Proposition 3.2 which we will prove in the following section, when dealing with the cubic coupling.

For now, let $R>0,0 \leq a<b \leq L$, and let $T>0$ be such that (1.9) holds. Let $0<\delta<\min (T / 2,(b-a) / 2) / 2$ such that $(2.8)$ holds for $2 \delta$ (note that it also holds for $\delta$ ). Define

$$
\begin{gathered}
\mathcal{Q}_{\delta}:=[\delta, T-\delta] \times[a+\delta, b-\delta], \\
\mathcal{Q}_{2 \delta}:=[2 \delta, T-2 \delta] \times[a+2 \delta, b-2 \delta],
\end{gathered}
$$

and let $\mathcal{Q} \subset[0, T] \times[a, b]$ be a smooth closed set such that

$$
\mathcal{Q}_{\delta} \subset \stackrel{\circ}{\mathcal{Q}}
$$


Define the following nonempty open differential relation of order 2:

$$
\mathcal{A}=\left\{(u, v, h) \in\left(C^{2}(\mathcal{Q})\right)^{3} \mid \forall(t, x) \in \mathcal{Q}, \frac{\partial f_{2}}{\partial u}(u(t, x), v(t, x)) \neq 0\right\} .
$$

We define the following nonlinear differential operator $\mathscr{D}: C^{2}(\mathcal{Q})^{3} \rightarrow C^{0}(\mathcal{Q})^{2}$ of order $r=2$ :

$$
\mathscr{D}((u, v, h))=\left(\square_{\nu_{1}} u-f_{1}(u, v)-h, \square_{\nu_{2}} v-f_{2}(u, v)\right) \quad \forall(u, v, h) \in C^{2}(\mathcal{Q})^{3}
$$

and its differential at $(u, v, h) \in C^{2}([0, T] \times[0, L])^{3}$ :

$$
\begin{aligned}
& \mathscr{L}_{(u, v, h)}(\tilde{u}, \tilde{v}, \tilde{h})=\left(\square_{\nu_{1}} \tilde{u}-D f_{1}(u, v) \cdot(\tilde{u}, \tilde{v})-\tilde{h}, \square_{\nu_{2}} \tilde{v}-D f_{2}(u, v) \cdot(\tilde{u}, \tilde{v})\right) \\
& \quad \forall(\tilde{u}, \tilde{v}, \tilde{h}) \in C^{2}([0, T] \times[0, L])^{3} .
\end{aligned}
$$

We now have the following result, thanks to the definition of $\mathcal{A}$.

Proposition 2.2. $\mathscr{D}$ admits an infinitesimal inversion of order 2 over $\mathcal{A}$.

Proof. Let $h_{1}, h_{2} \in C^{4}(\mathcal{Q}),(u, v, h) \in \mathcal{A}$. Using the fact that $\frac{\partial f_{2}}{\partial u}(u, v)$ never vanishes, if we set

$$
\begin{aligned}
& \tilde{v}=0, \\
& \tilde{u}=-\frac{h_{2}}{\frac{\partial f_{2}}{\partial u}(u, v)}, \\
& \tilde{h}=\square_{\nu_{1}} \tilde{u}-\frac{\partial f_{1}}{\partial u}(u, v) \tilde{u}-h_{1},
\end{aligned}
$$

then we have

$$
\mathscr{L}_{(u, v, h)}(\tilde{u}, \tilde{v}, \tilde{h})=\left(h_{1}, h_{2}\right) .
$$

Moreover, the above formulae clearly show that $(u, v, h) \mapsto \mathscr{L}_{(u, v, h)}(\tilde{u}, \tilde{v}, \tilde{h})$ is a (nonlinear, $C^{\infty}$ with the usual topology of $C^{2}(\mathcal{Q})$ ) differential operator of order 2 on $C^{2}(\mathcal{Q})$, and $(u, v, h, \tilde{u}, \tilde{v}, \tilde{h}) \mapsto \mathscr{L}_{(u, v, h)}(\tilde{u}, \tilde{v}, \tilde{h})$ is also $C^{\infty}$.

We can now apply Theorem 2.1 with $d=2, s=2, r=2 \sigma_{0}=7, \nu=\delta / 2$. This yields a collection of open sets, which all contain 0 ,

$$
\mathcal{B}_{z} \subset\left(C^{9}(\mathcal{Q})\right)^{2}, z \in \mathcal{A}^{11},
$$

the open subset of $\left(C^{11}(\mathcal{Q})\right)^{3} \times\left(C^{9}(\mathcal{Q})\right)^{2}$

$$
\mathcal{B}=\bigcup_{z \in \mathcal{A}^{11}}\{z\} \times \mathcal{B}_{z}
$$

and the collection of operators

$$
\mathscr{D}_{z}^{-1}: \mathcal{B}_{z} \rightarrow \mathcal{A}, z \in \mathcal{A}^{11}
$$

Now, thanks to condition (1.10),

$$
\begin{gathered}
(0,0,0) \in \mathcal{A}, \\
\mathscr{D}(0,0,0)=(0,0),
\end{gathered}
$$


and

$$
((0,0,0),(0,0)) \in \mathcal{B},
$$

so that, thanks to the neighborhood property of Theorem 2.1 , there exists $\varepsilon>0$ such that

$$
\left(B_{C^{11}(\mathcal{Q})}((0,0,0), \varepsilon)\right)^{3} \times\left(B_{C^{9}(\mathcal{Q})}((0,0), \varepsilon)\right)^{2} \subset \mathcal{B}
$$

By the continuity property of Theorem 2.1 with $\eta_{1}=\sigma_{1}=\sigma_{0}=7$, there exists $\eta>0$ such that for $\left\|\left((u, v, h),\left(h_{1}, h_{2}\right)\right)\right\|_{\left(C^{11}\right)^{3} \times\left(C^{9}\right)^{2}} \leq \eta$,

$$
\left\|\mathscr{D}_{(u, v, h)}^{-1}\left(h_{1}, h_{2}\right)\right\|_{\left(C^{6}\right)^{3}} \leq R .
$$

Proposition 2.1 with $k=11$ yields $\eta^{\prime}>0$ such that for any initial and final conditions

$$
\begin{aligned}
\left(\left(u_{0}, u_{1}\right),\left(v_{0}, v_{1}\right),\left(u_{0}^{f}, u_{1}^{f}\right),\left(v_{0}^{f}, v_{1}^{f}\right)\right) \in & \left(B_{C^{11}([0, T] \times[0, L])}\left(0, \eta^{\prime}\right)\right. \\
& \left.\times B_{C^{10}([0, T] \times[0, L])}\left(0, \eta^{\prime}\right)\right)^{4}
\end{aligned}
$$

there exist two controls $h_{1}, h_{2} \in C^{9}([0, T] \times[0, L])$, supported in $\mathcal{Q}_{2 \delta}$ (condition $\left.(2.9)\right)$, that steer system (2.7) from the given initial conditions to the given final conditions, with the corresponding trajectory $\left(u^{*}, v^{*}\right)$ satisfying (2.11). Together with (2.12), this implies that there exists $\eta^{\prime} \geq \eta^{\prime \prime}>0$ such that for initial and final conditions

$$
\begin{aligned}
\left(\left(u_{0}, u_{1}\right),\left(v_{0}, v_{1}\right),\left(u_{0}^{f}, u_{1}^{f}\right),\left(v_{0}^{f}, v_{1}^{f}\right)\right) \in & \left(B_{C^{11}([0, T] \times[0, L])}\left(0, \eta^{\prime \prime}\right)\right. \\
& \left.\times B_{C^{10}([0, T] \times[0, L])}\left(0, \eta^{\prime \prime}\right)\right)^{4},
\end{aligned}
$$

the corresponding trajectory of system (2.7) satisfies

$$
\begin{gathered}
\mathscr{D}\left(u_{\mid \mathcal{Q}}^{*}, v_{\mid \mathcal{Q}}^{*}, 0\right)=\left(h_{1 \mid \mathcal{Q}}, h_{2 \mid \mathcal{Q}}\right), \\
\left(\left(u_{\mid \mathcal{Q}}^{*}, v_{\mid \mathcal{Q}}^{*}, 0\right),\left(-h_{1 \mid \mathcal{Q}},-h_{2 \mid \mathcal{Q}}\right)\right) \in \mathcal{B}, \\
\left\|\left(\left(u^{*}, v^{*}, 0\right),\left(h_{1}, h_{2}\right)\right)\right\|_{\left(C^{11}\right)^{3} \times\left(C^{9}\right)^{2}} \leq \min (R, \eta) .
\end{gathered}
$$

Let us now set, keeping in mind the regularity property of Theorem 2.1 with $\eta_{1}=\sigma_{1}=\sigma_{0}=7$,

$$
(u, v, h)=\mathscr{D}_{\left(u_{\mid \mathcal{Q}}^{*}, v_{\mid \mathcal{Q}}^{*}, 0\right)}^{-1}\left(-h_{1 \mid \mathcal{Q}},-h_{2 \mid \mathcal{Q}}\right) \in \mathcal{A}^{6}
$$

Then, by the inversion property of Theorem 2.1, and (2.13),

$$
\mathscr{D}(u, v, h)=\mathscr{D}\left(u_{\mid \mathcal{Q}}^{*}, v_{\mid \mathcal{Q}}^{*}, 0\right)-\left(h_{1 \mid \mathcal{Q}}, h_{2 \mid \mathcal{Q}}\right)=(0,0)
$$

Now, let us show that $(u, v, h)=\left(u^{*}, v^{*}, 0\right)$ on $\stackrel{\mathcal{Q}}{\backslash} \mathcal{Q}_{\delta^{\prime}}$. This will allow us to extend $(u, v, h)$ on $([0, T] \times[0, L]) \backslash \mathcal{Q}$.

Let $(t, x) \in \mathcal{Q} \backslash \mathcal{Q}_{\delta}$. As the $h_{i}$ are supported in $\mathcal{Q}_{2 \delta}$,

$$
\left(\left(u^{*}, v^{*}, 0\right),\left(-h_{1},-h_{2}\right)\right)=\left(\left(u^{*}, v^{*}, 0\right),(0,0)\right) \quad \text { on } B\left((t, x), \frac{\delta}{2}\right) \cap \mathcal{Q} \text {. }
$$




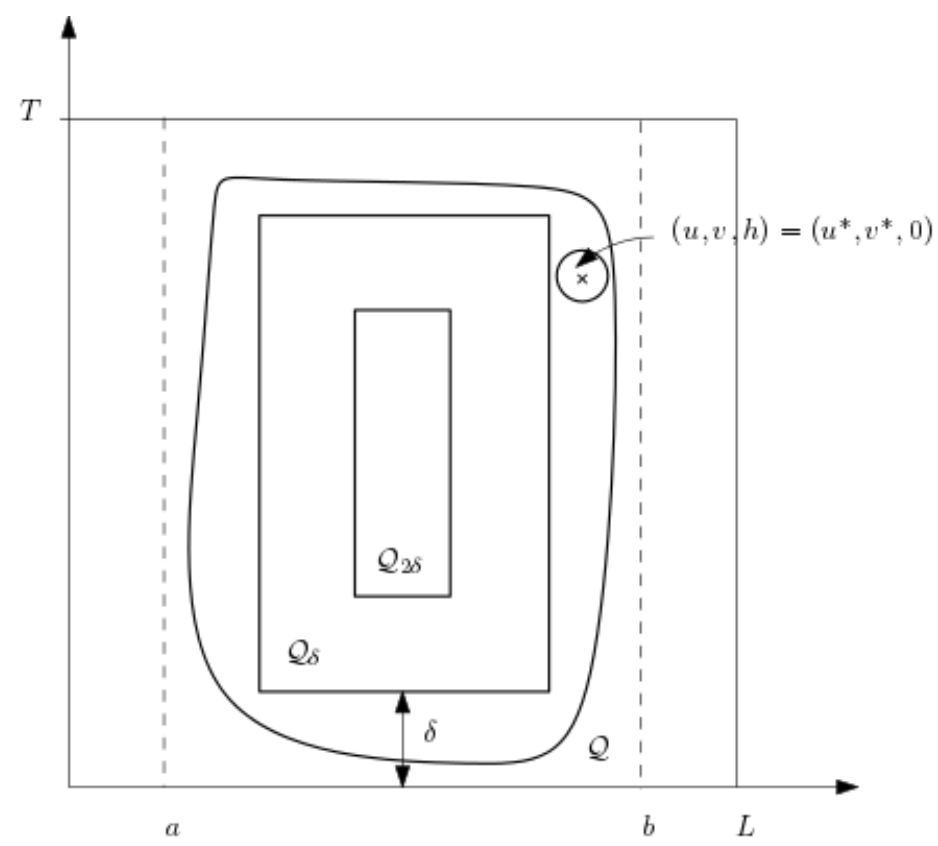

FIG. 1. Matching trajectories with two controls and with a single control on the appropriate domain.

Thus, using the locality property of Theorem 2.1,

$$
\mathscr{D}_{\left(u_{\mid \mathcal{Q}}^{*}, v_{\mid \mathcal{Q}}^{*}, 0\right)}^{-1}\left(-h_{1 \mid \mathcal{Q}},-h_{2 \mid \mathcal{Q}}\right)(t, x)=\mathscr{D}_{\left(u_{\mid \mathcal{Q}}^{*}, v_{\mid \mathcal{Q}}^{*}, 0\right)}^{-1}(0,0)(t, x),
$$

that is, using the normalization property,

$$
(u, v, h)(t, x)=\left(u^{*}, v^{*}, 0\right)(t, x) .
$$

We can now extend (see Figure 1) $(u, v, h)$ by setting

$$
(u, v, h)(t, x)=\left(u^{*}, v^{*}, 0\right)(t, x) \quad \forall(t, x) \in[0, T] \times[0, L] \backslash \mathcal{Q} .
$$

Then,

$$
\operatorname{supp} h \subset[0, T] \times[a, b],
$$

and $(u, v)$ satisfies the same initial, boundary, and final conditions as $\left(u^{*}, v^{*}\right)$ :

$$
\begin{aligned}
& \begin{cases}(u, v)(0, \cdot)=\left(u_{0}, v_{0}\right), & \left(u_{t}, v_{t}\right)(0, \cdot)=\left(u_{1}, v_{1}\right), \\
(u, v)(T, \cdot)=\left(u_{0}^{f}, v_{0}^{f}\right), & \left(u_{t}, v_{t}\right)(T, \cdot)=\left(u_{1}^{f}, v_{1}^{f}\right),\end{cases} \\
& \left\{\begin{array}{l}
u(\cdot, 0)=u(\cdot, L)=0, \\
v(\cdot, 0)=v(\cdot, L)=0,
\end{array}\right.
\end{aligned}
$$

and

$$
\left\{\begin{array}{l}
\square_{\nu_{1}} u=f_{1}(u, v)+h, \\
\square_{\nu_{2}} v=f_{2}(u, v),
\end{array}\right.
$$

Finally, we get (1.12) from (2.15) and the continuity property of Theorem 2.1. This proves Theorem 1.1. 
Remark 2.2. Theorem 1.1 actually holds for coupled quasi-linear equations:

$$
\left\{\begin{aligned}
\partial_{t t} u-\partial_{x}\left(K_{1}\left(u, \partial_{x} u\right)\right) & =f_{1}(u, v)+h, x \in[0, L], \\
\partial_{t t} v-\partial_{x}\left(K_{2}\left(v, \partial_{x} v\right)\right) & =f_{2}(u, v), x \in[0, L], \\
u & =0 \text { on }\{0, L\}, \\
v & =0 \text { on }\{0, L\},
\end{aligned}\right.
$$

where $f_{1}(0,0)=f_{2}(0,0)=0, K_{1}, K_{2} \in C^{\infty}\left(\mathbb{R}^{2}\right)$, and $K_{1}(0,0)=K_{2}(0,0)=0$. One can check that when one modifies the recurrence relation in (1.5) to match the new equations, the operators can still be written using only $J_{x}^{n}(u, v), J_{x}^{n-1}\left(u_{t}, v_{t}\right)$, and $J_{t}^{n}(u, v)$, and thus the compatibility conditions will have the same form as (1.8).

Indeed, in this case we can still use Li's results for the perturbed quasi-linear system, as we consider the "perturbations" around 0 . This will yield a "universal" time condition, because the propagation speeds are close to $\min \left(\sqrt{\partial_{2} K_{1}(0,0)}, \sqrt{\partial_{2} K_{2}(0,0)}\right)$ for the perturbed system. On the other hand if we work around a nonzero trajectory (return method), the perturbed quasi-linear system could present much smaller propagation speeds. The final time condition would then depend on the return trajectories that are found.

TheOREM 2.2. Let $R>0,0 \leq a<b \leq L, T>0$ such that

$$
\begin{aligned}
& T>2(L-b) \max \left(\left(\sqrt{\partial_{2} K_{1}(0,0)}\right)^{-1},\left(\sqrt{\partial_{2} K_{2}(0,0)}\right)^{-1}\right), \\
& T>2 a \max \left(\left(\sqrt{\partial_{2} K_{1}(0,0)}\right)^{-1},\left(\sqrt{\partial_{2} K_{2}(0,0)}\right)^{-1}\right) .
\end{aligned}
$$

If

$$
\frac{\partial f_{2}}{\partial u}(0,0) \neq 0
$$

then there exists $\eta>0$ such that for initial and final conditions

$$
\left(\left(u_{0}, u_{1}\right),\left(v_{0}, v_{1}\right),\left(u_{0}^{f}, u_{1}^{f}\right),\left(v_{0}^{f}, v_{1}^{f}\right)\right) \in B_{\left(C^{11}([0, L]) \times C^{10}([0, L])\right)^{4}}(0, \eta)
$$

compatible at the order 11 , there exists $h \in C^{6}([0, T] \times[0, L])$ such that

$$
\operatorname{supp} h \subset[0, T] \times[a, b],
$$

and such that the corresponding solution $(u, v) \in C^{6}([0, T] \times[0, L])$ of $(2.23)$ with initial values $\left(\left(u_{0}, u_{1}\right),\left(v_{0}, v_{1}\right)\right)$ satisfies

$$
\begin{cases}u(T, \cdot)=u_{0}^{f}, & u_{t}(T, \cdot)=u_{1}^{f}, \\ v(T, \cdot)=v_{0}^{f}, & v_{t}(T, \cdot)=v_{1}^{f},\end{cases}
$$

and inequality (1.12) holds.

3. Second case: An example with an uncontrollable linearized system. We now turn to system (1.2). As mentioned before, it does not satisfy condition (1.10); in other words, the linearized system around 0 is not controllable. Indeed, for

$$
\left\{\begin{array}{l}
\square_{\nu_{1}} u=h, \\
\square_{\nu_{2}} v=0, \\
u_{\mid \partial \Omega}=0, \\
v_{\mid \partial \Omega}=0,
\end{array}\right.
$$

the control $h$ gives us no influence on the dynamics of $v$. 
Thus, the computations from the beginning of subsection 2.2 do not hold: we cannot work around the stationary trajectory 0 , and thus we need to find another trajectory around which to work. More precisely, keeping in mind Proposition 2.2, we look for a return trajectory $(\bar{u}, \bar{v}, \bar{h})$ going from 0 to 0 such that for some smooth closed set $\mathcal{Q} \subset[0, T] \times[a, b]$, we have

$$
\forall(t, x) \in \mathcal{Q}, \frac{\partial f_{2}}{\partial u}(\bar{u}(t, x), \bar{v}(t, x))=3 \bar{u}^{2}(t, x) \neq 0 .
$$

Additionally, $\mathcal{Q}$ will have to satisfy some properties so that a result with two controls can be proved.

To find such a trajectory, we follow the same idea as in [CGR10], where return trajectories are built for coupled heat equations with a cubic coupling. The additional derivative in time simply adds terms and makes for heavier computations. However, condition (3.2) will account for additional work.

We will then prove and use a more general controllability result with two controls. After that, the application of Gromov's theorem is rather straightforward.

3.1. A preliminary construction: Elementary trajectories. In this subsection, we describe a construction of a smooth trajectory of system (1.2) that goes from 0 to 0 . For now we consider condition (3.2) but without any special requirements for $\mathcal{Q}$.

In what follows, we suppose without loss of generality (by scaling the space variable) that $\nu_{2}=1$.

To build trajectories that start at 0 and return there, the idea is to use the cascade structure of the equation: first we find a $C^{\infty}([-1,1] \times[0,1])$ function $\bar{v}$ such that $\square \bar{v}$ is the third power of a $C^{\infty}([-1,1] \times[0,1])$ function $\bar{u}$. By setting the right conditions at the start and end times, this gives us a return trajectory. The corresponding control will then be $\square_{\nu_{1}} \bar{u}$.

Let us recall that $x \mapsto \sqrt[3]{x}$ is $C^{\infty}$ on $\mathbb{R}^{*}$. So, by composition, the cubic root of a $C^{\infty}$ function $f$ is $C^{\infty}$ at all the points where $f$ is nonzero. At the points where $f$ vanishes, by Taylor's formula, a fairly simple sufficient condition for $\sqrt[3]{f}$ to be $C^{\infty}$ at those points is for $f$ to vanish, along with its first and second derivatives, while its third derivative is nonzero.

Now, to find functions whose image by the wave operator is a third power of a $C^{\infty}$ function, we consider the solutions to the corresponding stationary problem, namely, functions whose Laplacian is the third power of a $C^{\infty}$ function. The solution of this problem corresponds to the following proposition, proven (with $1 / 2$ instead of $3 / 4$ ) in [CGR10].

Proposition 3.1 (Coron, Guerrero, Rosier). There exist $\delta^{\prime}, \delta^{\prime \prime}, g \in C^{\infty}([0,1]), G \in$ $C^{\infty}([0,1])$ such that

$$
\left\{\begin{array}{l}
g^{\prime \prime}=G, \\
g(z)=1-z^{2} \text { on }\left[0, \delta^{\prime \prime}\right], \\
g(z)=e^{-\frac{1}{1-z^{2}}} \text { on }\left[1-\delta^{\prime}, 1\right), \\
G(z)\left(z-\frac{3}{4}\right)>0 \text { for } z \in(0,1) \backslash\left\{\frac{3}{4}\right\}, \\
G(z)=\left(z-\frac{3}{4}\right)^{3} \text { on }\left[\frac{3}{4}-\frac{\delta^{\prime \prime}}{2}, \frac{3}{4}+\frac{\delta^{\prime \prime}}{2}\right] .
\end{array}\right.
$$

In a sense, this proposition gives us the simplest example of functions the second derivative of which is the third power of a smooth function: $G=g^{\prime \prime}$ vanishes 
exponentially in 1 and has only one vanishing point on $[0,1)$, around which it has a cubic behavior. The idea of the construction is then to perturb this function of space and make it evolve in time, so slightly as to preserve the properties (3.3) of the stationary problem. Let $0 \leq a<b \leq L$, and $T>0$ such that (1.9) holds.

Let $0<\delta<\min (T / 2,(b-a) / 2)$ such that (3.4) holds. Set $\lambda_{0}$ to be a function such that

$$
\begin{aligned}
& \begin{array}{c}
\lambda_{0}(t)=e^{-\sqrt{\frac{1}{t(T-t)}}} \quad \forall t \in\left(0, \frac{\delta}{2}\right] \cup\left[T-\frac{\delta}{2}, T\right), \\
\lambda_{0}(0)=\lambda_{0}(T)=0,
\end{array} \\
& \lambda_{0}(t)>0 \quad \forall t \in(0, T), \\
& \lambda_{0}([\delta, T-\delta])=\{1\},
\end{aligned}
$$

and write $\lambda:=\varepsilon \lambda_{0}$ for some $\varepsilon$ to be determined.

Remark 3.1. In [CGR10], the authors take

$$
\lambda(t)=\varepsilon t^{2}(1-t)^{2} .
$$

In our case, however, we will see that we need to fit a rectangle of the form $[\delta, T-$ $\delta] \times\left[x_{0}-\xi, x_{0}+\xi\right]$ inside the support of $\bar{u}$ (see Figures 2 and 3 ). With a polynomial as in (3.5), the smaller $\delta>0$ gets, the smaller $\xi$ has to be. This in itself would not be an obstruction to prove our controllability result, but using definition (3.4) has the advantage to fix the width of the rectangle for all $\delta$ satisfying (2.8).

Set

$$
\begin{gathered}
f_{0}(t)=e^{-\frac{1}{t(t-T)}} \quad \forall t \in(0, T), \\
f_{0}(0)=f_{0}(T)=0 .
\end{gathered}
$$

Finally, let $g_{0}$ be the solution to the stationary problem (see Proposition 3.1). Let $x_{0} \in(0, L)$, and choose $\varepsilon \leq \min \left(x_{0}, L-x_{0}\right)$. We now look for $\bar{v}$ in the form

$$
\bar{v}(t, x)=\sum_{i=0}^{3} f_{i}(t) g_{i}\left(\frac{\left|x-x_{0}\right|}{\lambda(t)}\right) .
$$

Note that the fact that $f_{0}$ vanishes faster than $\lambda$ at 0 and $T$ compensates the singularity that occurs in the term $\left|x-x_{0}\right| / \lambda(t)$ of the first term of the sum. We will see that the $f_{i}$ have a similar property, thus ensuring that functions of the form above are indeed $C^{\infty}$. We also require that the $g_{i}$ satisfy

$$
\text { supp } g_{i} \subset\left[\frac{3}{4}-\frac{\delta^{\prime \prime}}{2}, \frac{3}{4}+\frac{\delta^{\prime \prime}}{2}\right] \forall i \in\{1,2,3\} \text {, }
$$

where $\delta^{\prime \prime}$ is as defined in Proposition 3.1, so that

$$
\operatorname{supp}(\bar{u}, \bar{v}, \bar{h}) \subset[0, T] \times\left[x_{0}-\varepsilon, x_{0}+\varepsilon\right] .
$$

Let us then set, in order to simplify the notation for our computations,

$$
\begin{gathered}
z:=\frac{\left|x-x_{0}\right|}{\lambda(t)}, \\
V(t, x):=\square \bar{v}=\bar{v}_{t t}-\bar{v}_{x x},
\end{gathered}
$$




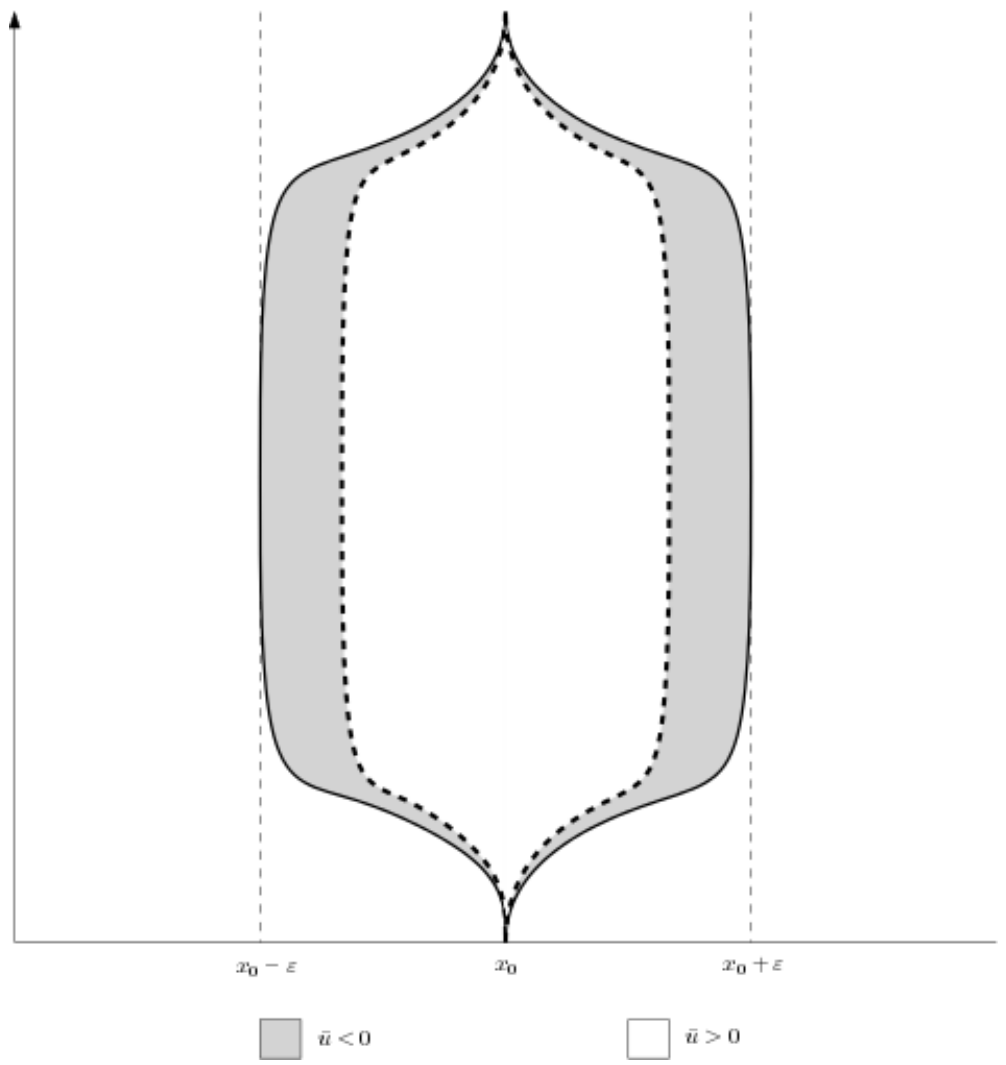

FIG. 2. The support of the trajectory $(\bar{u}, \bar{v}, \bar{h})$. The dashed line represents the vanishing points of $\square \bar{v}$ (or, equivalently, $\bar{u}$ ).

which we note, in the new set of variables,

$$
\mathcal{V}(t, z):=V(t, \lambda(t) z)
$$

We are now looking for functions $f_{i}$ and $g_{i}$ such that $V^{\frac{1}{3}}$ is of class $C^{\infty}$. In order to achieve this, we will work with the new set of variables $(t, z)$ and study $\mathcal{V}$. We now need to have precise knowledge of the behavior of $\mathcal{V}$ when it vanishes.

More precisely, the aim is to write $\mathcal{V}$ near $\frac{3}{4}$ as

$\lambda^{2} \mathcal{V}=\left(z-\frac{3}{4}\right)^{3} \varphi(t, z)$ with $\varphi \in C^{\infty}\left([0, T] \times\left[\frac{3}{4}-\frac{\delta^{\prime \prime}}{2}, \frac{3}{4}+\frac{\delta^{\prime \prime}}{2}\right]\right), \varphi<0$ for $t \neq 0, T$.

Note that $\varphi$ has to be negative because of the minus sign in the wave operator. Hence, we look for $\mathcal{V}$ satisfying

$$
\begin{gathered}
\mathcal{V}_{z}\left(\cdot, \frac{3}{4}\right)=0 \\
\mathcal{V}_{z z}\left(\cdot, \frac{3}{4}\right)=0 \\
\mathcal{V}_{z z z} \leq-C f_{0} \text { on }[0, T] \times\left[\frac{3}{4}-\frac{\delta^{\prime \prime}}{2}, \frac{3}{4}+\frac{\delta^{\prime \prime}}{2}\right], \text { where } C>0
\end{gathered}
$$


Additionally, since we have the condition on $G$

$$
G(z)\left(z-\frac{3}{4}\right)>0 \text { for } z \in(0,1) \backslash\left\{\frac{3}{4}\right\},
$$

we will make sure to have

$$
\mathcal{V}(t, z)\left(z-\frac{3}{4}\right)<0 \quad \forall(t, z) \in(0, T) \times\left((0,1) \backslash\left\{\frac{3}{4}\right\}\right) .
$$

Let us now compute $\mathcal{V}$ and its first, second, and third derivatives:

$$
\begin{aligned}
\bar{v}_{t t}=\sum_{i=0}^{3} \ddot{f}_{i} g_{i}-2 \dot{f}_{i} z \frac{\dot{\lambda}}{\lambda} g_{i}^{\prime}-f_{i}\left(z \frac{\lambda \ddot{\lambda}-2 \dot{\lambda}^{2}}{\lambda^{2}} g_{i}^{\prime}-z^{2}\left(\frac{\dot{\lambda}}{\lambda}\right)^{2} g_{i}^{\prime \prime}\right), \\
\bar{v}_{x x}=\lambda^{-2} f_{0} G+\sum_{i=1}^{3} f_{i} \lambda^{-2} g_{i}^{\prime \prime}, \\
\lambda^{2} \mathcal{V}=-\left(1-z^{2} \dot{\lambda}^{2}\right) f_{0} G+\lambda^{2} \ddot{f}_{0} g_{0}-2 \dot{f}_{0} z \dot{\lambda} \lambda g_{0}^{\prime}-z\left(\lambda \ddot{\lambda}-2 \dot{\lambda}^{2}\right) f_{0} g_{0}^{\prime} \\
\quad+\sum_{i=1}^{3}\left(\lambda^{2} \ddot{f}_{i} g_{i}-2 \dot{f}_{i} z \dot{\lambda} \lambda g_{i}^{\prime}-f_{i}\left(z\left(\lambda \ddot{\lambda}-2 \dot{\lambda}^{2}\right) g_{i}^{\prime}-z^{2} \dot{\lambda}^{2} g_{i}^{\prime \prime}\right)-f_{i} g_{i}^{\prime \prime}\right) \\
=-\left(1-z^{2} \dot{\lambda}^{2}\right) f_{0} G+\lambda^{2} \ddot{f}_{0} g_{0}-2 \dot{f}_{0} z \dot{\lambda} \lambda g_{0}^{\prime}-z\left(\lambda \ddot{\lambda}-2 \dot{\lambda}^{2}\right) f_{0} g_{0}^{\prime} \\
\quad+\sum_{i=1}^{3}\left(\lambda^{2} \ddot{f}_{i} g_{i}-2 \dot{f}_{i} z \dot{\lambda} \lambda g_{i}^{\prime}-f_{i}\left[z\left(\lambda \ddot{\lambda}-2 \dot{\lambda}^{2}\right) g_{i}^{\prime}+\left(1-z^{2} \dot{\lambda}^{2}\right) g_{i}^{\prime \prime}\right]\right) .
\end{aligned}
$$

Now, for $\varepsilon$ small enough (note that this depends on the value of $\delta$ ),

$$
1-\left(\varepsilon z \dot{\lambda_{0}}(t)\right)^{2}>\frac{1}{2} \quad \forall(t, z) \in[0, T] \times[0,1]
$$

and, using the notation $\lambda$,

$$
\left\|\frac{1}{1-\left(\frac{3}{4} \dot{\lambda}\right)^{2}}\right\|_{C^{2}([0, T])} \leq 10 .
$$

Now, if we impose

$$
\left\{\begin{array}{l}
g_{i}^{(j)}\left(\frac{3}{4}\right)=0 \forall i \in\{1,2,3\}, j \in\{0,1,2\}(i, j) \neq(1,2) \\
g_{1}^{(2)}\left(\frac{3}{4}\right)=1
\end{array}\right.
$$

and if we define $f_{1}$ by

$$
f_{1}:=\frac{1}{1-\left(\frac{3}{4} \dot{\lambda}\right)^{2}}\left(\lambda^{2} g_{0}\left(\frac{3}{4}\right) \ddot{f}_{0}-2 \frac{3}{4} \dot{\lambda} \lambda g_{0}^{\prime}\left(\frac{3}{4}\right) \dot{f}_{0}-\frac{3}{4}\left(\lambda \ddot{\lambda}-2 \dot{\lambda}^{2}\right) g_{0}^{\prime}\left(\frac{3}{4}\right) f_{0}\right),
$$

we get

$$
\lambda^{2} \mathcal{V}\left(\cdot, \frac{3}{4}\right)=0
$$


We now compute the first derivative of $\mathcal{V}$ :

$$
\begin{aligned}
\lambda^{2} \mathcal{V}_{z}= & -\left(1-z^{2} \dot{\lambda}^{2}\right) f_{0} G^{\prime}+\left(2 z \dot{\lambda}^{2}-z\left(\lambda \ddot{\lambda}-2 \dot{\lambda}^{2}\right)\right) f_{0} G \\
& -2 z \dot{\lambda} \lambda \dot{f}_{0} G+\lambda^{2} \ddot{f}_{0} g_{0}^{\prime}-2 \dot{f_{0}} \dot{\lambda} \lambda g_{0}^{\prime}-\left(\lambda \ddot{\lambda}-2 \dot{\lambda}^{2}\right) f_{0} g_{0}^{\prime}+\sum_{i=1}^{3} \lambda^{2} \ddot{f}_{i} g_{i}^{\prime}-2 \dot{f}_{i} z \dot{\lambda} \lambda g_{i}^{\prime \prime} \\
& -2 \dot{f_{i}} \dot{\lambda} \lambda g_{i}^{\prime}-f_{i}\left[\left(\lambda \ddot{\lambda}-2 \dot{\lambda}^{2}\right) g_{i}^{\prime}-2 z \dot{\lambda}^{2} g_{i}^{\prime \prime}\right]-f_{i}\left[z\left(\lambda \ddot{\lambda}-2 \dot{\lambda}^{2}\right) g_{i}^{\prime \prime}+\left(1-z^{2} \dot{\lambda}^{2}\right) g_{i}^{(3)}\right] \\
= & -\left(1-z^{2} \dot{\lambda}^{2}\right) f_{0} G^{\prime}+\left(4 z \dot{\lambda}^{2}+z \lambda \ddot{\lambda}\right) f_{0} G \\
& -2 z \dot{\lambda} \lambda \dot{f}_{0} G+\lambda^{2} \ddot{f}_{0} g_{0}^{\prime}-2 \dot{f}_{0} \dot{\lambda} \lambda g_{0}^{\prime}-\left(\lambda \ddot{\lambda}-2 \dot{\lambda}^{2}\right) f_{0} g_{0}^{\prime} \\
& +\sum_{i=1}^{3} \lambda^{2} \ddot{f}_{i} g_{i}^{\prime}-2 \dot{f}_{i} \dot{\lambda} \lambda\left(z g_{i}^{\prime \prime}+g_{i}^{\prime}\right) \\
& -f_{i}\left[\left(\lambda \ddot{\lambda}-2 \dot{\lambda}^{2}\right) g_{i}^{\prime}+z\left(\lambda \ddot{\lambda}-4 \dot{\lambda}^{2}\right) g_{i}^{\prime \prime}+\left(1-z^{2} \dot{\lambda}^{2}\right) g_{i}^{(3)}\right] .
\end{aligned}
$$

Again, we impose

$$
g_{i}^{(3)}\left(\frac{3}{4}\right)=\left\{\begin{array}{l}
0 \text { if } i \in\{1,3\} \\
1 \text { if } i=2
\end{array}\right.
$$

and we set

$$
\begin{aligned}
f_{2}:= & \frac{1}{1-\left(\frac{3}{4} \dot{\lambda}\right)^{2}}\left[\lambda^{2} g_{0}^{\prime}\left(\frac{3}{4}\right) \ddot{f}_{0}-\dot{\lambda} \lambda g_{0}^{\prime}\left(\frac{3}{4}\right) \dot{f}_{0}-2 \dot{\lambda} \lambda g_{0}\left(\frac{3}{4}\right) \dot{f}_{0}\right. \\
& \left.-\left(\lambda \ddot{\lambda}-2 \dot{\lambda}^{2}\right) g_{0}^{\prime}\left(\frac{3}{4}\right) f_{0}-2 \frac{3}{4} \dot{\lambda} \lambda \dot{f}_{1}-\frac{3}{4}\left(\lambda \ddot{\lambda}-4 \dot{\lambda}^{2}\right) f_{1}\right]
\end{aligned}
$$

so that

$$
\lambda^{2} \mathcal{V}_{z}\left(\cdot, \frac{3}{4}\right)=0
$$

Finally,

$$
\begin{aligned}
\lambda^{2} \mathcal{V}_{z z}= & -\left(1-z^{2} \dot{\lambda}^{2}\right) f_{0} G^{\prime \prime}+\left(6 z \dot{\lambda}^{2}+z \lambda \ddot{\lambda}\right) f_{0} G^{\prime} \\
& -2 z \dot{\lambda} \lambda \dot{f}_{0} G^{\prime}+6 \dot{\lambda}^{2} f_{0} G-4 \dot{\lambda} \lambda \dot{f_{0}} G+\lambda^{2} \ddot{f}_{0} G \\
& +\sum_{i=1}^{3} \lambda^{2} \ddot{f}_{i} g_{i}^{\prime \prime}-2 \dot{f_{i}} \dot{\lambda} \lambda\left(2 g_{i}^{\prime \prime}+z g_{i}^{(3)}\right) \\
& -f_{i}\left[\left(2 \lambda \ddot{\lambda}-6 \dot{\lambda}^{2}\right) g_{i}^{\prime \prime}+z\left(\lambda \ddot{\lambda}-6 \dot{\lambda}^{2}\right) g_{i}^{(3)}+\left(1-z^{2} \dot{\lambda}^{2}\right) g_{i}^{(4)}\right] .
\end{aligned}
$$

Again we impose

$$
g_{i}^{(4)}\left(\frac{3}{4}\right)=\left\{\begin{array}{l}
0 \text { if } i \in\{1,2\} \\
1 \text { if } i=3
\end{array}\right.
$$

then, by setting

$$
f_{3}=\frac{1}{1-\left(\frac{3}{4} \dot{\lambda}\right)^{2}}\left[-\left(2 \lambda \dot{\lambda}+2 \dot{\lambda}^{2}\right) f_{1}-4 \lambda \dot{\lambda} \dot{f}_{1}+\lambda^{2} \ddot{f}_{1}-\frac{3}{4}\left(\lambda \ddot{\lambda}-6 \dot{\lambda}^{2}\right) f_{2}-2 \frac{3}{4} \dot{\lambda} \lambda \dot{f}_{2}\right],
$$

we get

$$
\lambda^{2} \mathcal{V}_{z z}\left(\cdot, \frac{3}{4}\right)=0
$$


Now all that remains is to estimate the third derivative: on $[0, T] \times\left[\frac{3}{4}-\frac{\delta^{\prime \prime}}{2}, \frac{3}{4}+\frac{\delta^{\prime \prime}}{2}\right]$, by definition of $G$, we have

$$
\lambda^{2} \mathcal{V}_{z z z}=-6 K_{\frac{3}{4}}\left(1-z^{2} \dot{\lambda}^{2}\right) f_{0}+\mathcal{R}_{0}+\mathcal{R}
$$

with

$$
\mathcal{R}_{0}:=z\left(8 \dot{\lambda}^{2}+\ddot{\lambda} \lambda\right) f_{0} G^{\prime \prime}-2 z \dot{\lambda} \lambda \dot{f}_{0} G^{\prime \prime}+\left(12 \dot{\lambda}^{2}+\lambda \ddot{\lambda}\right) f_{0} G^{\prime}-6 \dot{\lambda} \lambda \dot{f}_{0} G^{\prime}+\lambda^{2} \ddot{f}_{0} G^{\prime}
$$

and

$$
\begin{aligned}
\mathcal{R}:= & \sum_{i=1}^{3} \lambda^{2} \ddot{f}_{i} g_{i}^{(3)}-2 \dot{f}_{i} \dot{\lambda} \lambda\left(3 g_{i}^{(3)}+z g_{i}^{(4)}\right) \\
& -f_{i}\left[\left(3 \ddot{\lambda} \lambda-12 \dot{\lambda}^{2}\right) g_{i}^{(3)}+\left(z \lambda \ddot{\lambda}-8 z \dot{\lambda}^{2}\right) g_{i}^{(4)}+\left(1-z^{2} \dot{\lambda}^{2}\right) g_{i}^{(5)}\right] .
\end{aligned}
$$

Let us note that (3.6), combined with the properties of exponential functions, yields

$$
\left(\frac{d}{d t}\right)^{n} f_{0}=F_{n}(t) f_{0}(t) \quad \forall n \in \mathbb{N},
$$

where the $F_{n}$ are rational fractions, the poles of which are 0 and $T$. Now, one can see in (3.19), (3.13), (3.15), and (3.17) that the divergent behavior of these fractions near 0 and $T$ is always compensated by the exponential behavior of $\lambda$ and its derivatives. Furthermore, differentiating the $f_{i}$ does not change this fact. Hence, keeping (3.11) in mind,

$$
\begin{aligned}
\mathcal{R}_{0} & =\varepsilon^{2} \mathcal{O}(f ; t, z), \\
f_{1}^{(n)} & =\varepsilon^{2} \mathcal{O}\left(f_{0} ; t\right) \quad \forall n \in \mathbb{N}, \\
f_{2}^{(n)} & =\varepsilon^{2} \mathcal{O}\left(f_{0} ; t\right) \quad \forall n \in \mathbb{N}, \\
f_{3}^{(n)} & =\varepsilon^{4} \mathcal{O}\left(f_{0} ; t\right) \quad \forall n \in \mathbb{N},
\end{aligned}
$$

where the notation $\mathcal{O}(f ; t)$ (resp., $\mathcal{O}(f ; t, z)$ ) means $f$ times a bounded function of time on $[0, T]$ (resp., time and space). Hence, near $\frac{3}{4} A$, we have

$$
\mathcal{R}_{0}+\mathcal{R}=\varepsilon^{2} \mathcal{O}\left(f_{0} ; t, z\right),
$$

the dominant term being $\ddot{f}_{1}\left(1-z^{2} \dot{\lambda}^{2}\right) g_{i}^{(5)}$. Consequently, using (3.18) and (3.23), for a small enough $\varepsilon$, there exists a constant $C>0$ such that

$$
\lambda^{2} \mathcal{V}_{z z z} \leq-C f_{0} \text { on }\left[\frac{3}{4}-\frac{\delta^{\prime \prime}}{2}, \frac{3}{4}+\frac{\delta^{\prime \prime}}{2}\right] .
$$

Thus, on $[0, T] \times\left[\frac{3}{4}-\frac{\delta^{\prime \prime}}{2}, \frac{3}{4}+\frac{\delta^{\prime \prime}}{2}\right]$, we can write, thanks to the Taylor-Laplace formula:

$$
\lambda^{2} \mathcal{V}=\left(z-\frac{3}{4}\right)^{3} \varphi(t, z) \text { with } \varphi \in C^{\infty}\left([0, T] \times\left[\frac{3}{4}-\frac{\delta^{\prime \prime}}{2}, \frac{3}{4}+\frac{\delta^{\prime \prime}}{2}\right]\right), \varphi<0 \text { for } t \neq 0, T
$$

Additionally, by the definition of $f_{0}, \varphi / \lambda^{2}$ vanishes exponentially for $t=0, T$, and (3.8) ensures that $\varphi$ vanishes exponentially for $z=1$, so that

$$
\left(\frac{\varphi}{\lambda^{2}}\right)^{\frac{1}{3}} \in C^{\infty}\left([0, T] \times\left[\frac{3}{4}-\frac{\delta^{\prime \prime}}{2}, \frac{3}{4}+\frac{\delta^{\prime \prime}}{2}\right]\right) .
$$


We now have

$$
\mathcal{V}^{\frac{1}{3}} \in C^{\infty}\left([0, T] \times\left[\frac{3}{4}-\frac{\delta^{\prime \prime}}{2}, \frac{3}{4}+\frac{\delta^{\prime \prime}}{2}\right]\right) .
$$

Moreover, on $[0, T] \times\left(\left[0, \frac{3}{4}-\frac{\delta^{\prime \prime}}{2}\right) \cup\left(\frac{3}{4}-\frac{\delta^{\prime \prime}}{2}, 1\right]\right)$, thanks to the constraint on the supports of the $g_{i}$, we have

$$
\lambda^{2} \mathcal{V}=-\left(1-z^{2} \dot{\lambda}^{2}\right) f_{0} G+\underbrace{\lambda^{2} \ddot{f}_{0} g_{0}-2 \dot{f}_{0} z \dot{\lambda} \lambda g_{0}^{\prime}-z\left(\lambda \ddot{\lambda}-2 \dot{\lambda}^{2}\right) f_{0} g_{0}^{\prime}}_{\varepsilon^{2} \mathcal{O}\left(f_{0} ; t, x\right)} .
$$

As, thanks to Proposition 3.1, we have

$$
|G|>2 \text { on }\left[0, \frac{3}{4}-\frac{\delta^{\prime \prime}}{2}\right] \cup\left[\frac{3}{4}+\frac{\delta^{\prime \prime}}{2}, 1-\delta^{\prime}\right],
$$

for small enough $\varepsilon$, we have

$$
\left.\left|\lambda^{2} \mathcal{V}\right|>0 \text { on }\right] 0, T\left[\times\left(\left[0, \frac{3}{4}-\frac{\delta^{\prime \prime}}{2}\right] \cup\left[\frac{3}{4}+\frac{\delta^{\prime \prime}}{2}, 1-\delta^{\prime}\right]\right) .\right.
$$

Now, let us recall that, on $\left[1-\delta^{\prime}, 1\right)$,

$$
\begin{aligned}
& g_{0}(z)=e^{-\frac{1}{1-z^{2}}} \\
& g_{0}^{\prime}(z)=\frac{-2 z}{\left(1-z^{2}\right)^{2}} e^{-\frac{1}{1-z^{2}}}, \\
& G(z)=g_{0}^{\prime \prime}(z)=\frac{6 z^{4}-2}{\left(1-z^{2}\right)^{4}} e^{-\frac{1}{1-z^{2}}},
\end{aligned}
$$

so that $g_{0} / G$ and $g_{0}^{\prime} / G$ are bounded near 1 , allowing us to write

$$
\lambda^{2} \mathcal{V}=-f_{0} G+\varepsilon^{2} \mathcal{O}\left(f_{0} ; t\right) \mathcal{O}_{1^{-}}(G ; z)
$$

The notation $\mathcal{O}_{1^{-}}(G ; z)$ means $G$ times a bounded function of space on $\left[1-\delta^{\prime}, 1\right]$. So for small enough $\varepsilon$, there exists a function $a$ with positive values on $] 0, T[$ such that

$$
\lambda^{2} \mathcal{V}(t, z) \leq-a(t) G(z)<0 \quad \forall(t, z) \in(-0, T) \times\left[1-\delta^{\prime}, 1\right) .
$$

Finally, for all $t \in[0, T], \mathcal{V}(t, \cdot)$ vanishes exponentially at $z=1$, and for all $z \in$ $\left[1-\delta^{\prime}, 1\right], \mathcal{V}(\cdot, z)$ vanishes exponentially for $t=0, T$. Hence,

$$
\mathcal{V}^{\frac{1}{3}} \in C^{\infty}\left([0, T] \times\left(\left[0, \frac{3}{4}-\frac{\delta^{\prime \prime}}{2}\right) \cup\left(\frac{3}{4}+\frac{\delta^{\prime \prime}}{2}, 1\right]\right)\right) .
$$

This, together with (3.25), proves that

$$
\mathcal{V}^{\frac{1}{3}} \in C^{\infty}([0, T] \times[0,1]) .
$$

Now, as $x \mapsto|x|$ is $C^{\infty}$ on $\mathbb{R} \backslash\{0\}$, by composition we deduce from (3.29) that

$$
V^{\frac{1}{3}} \in C^{\infty}\left([0, T] \times\left((0, L) \backslash\left\{x_{0}\right\}\right)\right) .
$$

To deal with the missing point $x_{0}$, let us recall that for all $\left.t \in\right]-1,1[$, for all $x \in[0, L]$ such that $\left|x-x_{0}\right| \leq \delta^{\prime \prime} \lambda(t)$ (i.e., $\left.z \leq \delta^{\prime \prime}\right)$, 


$$
\begin{aligned}
\lambda^{2} \mathcal{V}(t, z)= & -f_{0} G+\lambda^{2} \ddot{f}_{0} g_{0}-2 \dot{f}_{0} z \dot{\lambda} \lambda g_{0}^{\prime}-z\left(\lambda \ddot{\lambda}-2 \dot{\lambda}^{2}\right) f_{0} g_{0}^{\prime}+z^{2} \dot{\lambda}^{2} f_{0} G \\
= & 2 f_{0}+\ddot{f}_{0}\left(\lambda^{2}-\left|x-x_{0}\right|^{2}\right)+4 \dot{f_{0}} \frac{\dot{\lambda}}{\lambda}\left|x-x_{0}\right|^{2} \\
& +2\left(\frac{\ddot{\lambda}}{\lambda}-2\left(\frac{\dot{\lambda}}{\lambda}\right)^{2}\right) f_{0}\left|x-x_{0}\right|^{2}-2\left(\frac{\dot{\lambda}}{\lambda}\right)^{2} f_{0}\left|x-x_{0}\right|^{2} \\
= & 2 f_{0}+\lambda^{2} \ddot{f}_{0}+\psi(t)\left|x-x_{0}\right|^{2},
\end{aligned}
$$

where $\psi \in C^{\infty}([0, T])$, and $\psi$ vanishes exponentially for $t=0, T$, along with all its derivatives.

We now see that the terms in $\left|x-x_{0}\right|$ of $V$ are actually in $\left|x-x_{0}\right|^{2}$, which compensates the singularity at 0 of the map $x \mapsto|x|$. Thus, from the smoothness of $\mathcal{V}^{\frac{1}{3}}$ we get, by composition, $V^{\frac{1}{3}} \in C^{\infty}([0, T] \times[0, L])$. Thus we have proved that, by chosing $g_{i}$ that verify $(3.8),(3.12),(3.14)$, and (3.16), we get

$$
V^{\frac{1}{3}} \in C^{\infty}([0, T] \times[0, L]) .
$$

Finally, we set

$$
\begin{aligned}
\bar{v}(x, t) & :=\sum_{i=0}^{3} f_{i}(t) g_{i}\left(\frac{\left|x-x_{0}\right|}{\lambda(t)}\right), \\
\bar{u} & :=(\square \bar{v})^{\frac{1}{3}} \\
\bar{h} & :=\square \bar{u},
\end{aligned}
$$

where $\lambda$ is defined by (3.4), the $g_{i}$ are some functions satisfying (3.8), (3.12), (3.14), and (3.16), and the $f_{i}$ are defined by (3.6), (3.13), (3.15), and (3.17).

Let us check that we have indeed built a return trajectory: for $i \in\{0, \ldots, 3\}$, the $f_{i}$ vanish at -1 and 1 , along with all their derivatives. Hence,

$$
\begin{gathered}
\bar{u}(-1, \cdot)=\bar{v}(-1, \cdot)=\bar{u}_{t}(-1, \cdot)=\bar{v}_{t}(-1, \cdot)=0, \\
\bar{u}(1, \cdot)=\bar{v}(1, \cdot)=\bar{u}_{t}(1, \cdot)=\bar{v}_{t}(1, \cdot)=0 .
\end{gathered}
$$

Remark 3.2. Most of the work in the construction above comes from the vanishing points $(t,(3 / 4) \lambda(t))$ "in the middle of the domain," So one could wonder, would it not be simpler to try to build a function that only vanishes, along with all its derivatives, at the points $(t, \lambda(t))$ ?

Let us recall that our strategy to build the return trajectory is to start from a solution to the stationary problem and then make it evolve through time so as to stay "not too far away from it." But the reason we have vanishing points "in the middle of the domain" has to do with that same stationary problem. More precisely, the stationary problem consists in finding functions that vanish, along with their derivatives, on the boundary of the domain. In our case this condition corresponds to

$$
g(z)=e^{-\frac{1}{1-z^{2}}} \text { on }\left[1-\delta^{\prime}, 1\right] .
$$

We further require that the Laplacians of these functions be third powers of $C^{\infty}$ functions. In our case this condition becomes

$$
G(z)\left(z-\frac{3}{4}\right)>0
$$




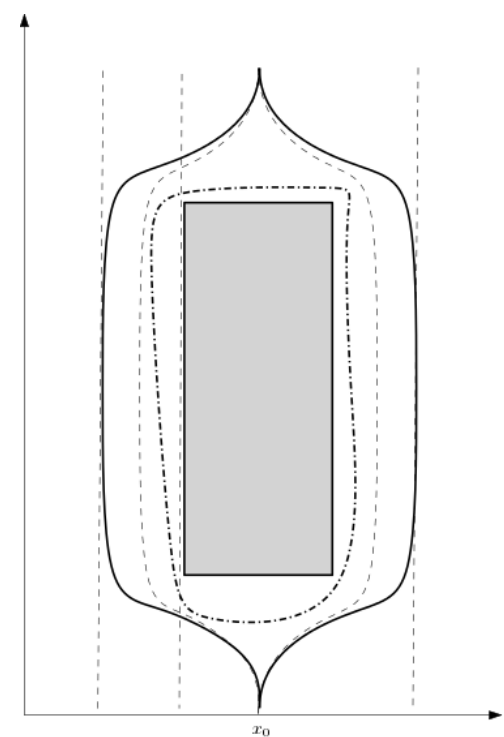

FIG. 3. The support of the preliminary construction with a rectangle fit inside the line of vanishing points of $\bar{u}$.

$$
G(z)=\left(z-\frac{3}{4}\right)^{3} \text { on }\left[\frac{3}{4}-\frac{\delta^{\prime \prime}}{2}, \frac{3}{4}+\frac{\delta^{\prime \prime}}{2}\right]
$$

Now, we could instead demand that $G$ be nonnegative (or nonpositive). But then, by convexity arguments (or Hopf's maximum principle), we would get

$$
g^{\prime}(1)<0
$$

which contradicts condition (3.31). But that condition is very helpful in proving the smoothness of $\mathcal{V}^{\frac{1}{3}}$ near the boundary. Giving it up would mean setting more conditions on the $g_{i}$ functions near the boundary, so we would have to give up condition (3.8) and then set additional conditions on the $g_{i}$ to make sure $V$ is well defined (as $\lambda(0)=$ $\lambda(T)=0$ ), preserve the sign of $\mathcal{V}$ or more generally its smoothness, in particular near the boundary ..., which would probably be more trouble than what we had to do at the vanishing points $(t,(3 / 4) \lambda(t))$.

3.2. Covering sets and return trajectories. As mentioned at the beginning of this section, we want to work on a smooth subset of $[0, T] \times[a, b]$, where $u \neq 0$. However, to do so we need more than the elementary trajectory described above: rather, we use the elementary trajectory as a building block for our final return trajectory. Indeed, let $0<\delta<\min ((b-a) / 4, T / 2)$ such that $(2.8)$ is satisfied. The preliminary construction gives us a real number $\varepsilon>0$ (after the right rescaling of the space variable) and, for any $x_{0} \in[a+\delta+\varepsilon, b-\delta-\varepsilon]$, a trajectory $(\bar{u}, \bar{v}, \bar{h})$ such that $\bar{u} \neq 0$ on $\Lambda_{\varepsilon, x_{0}}:=\left\{(t, x)|| x-x_{0} \mid<(3 / 4) \varepsilon \lambda_{0}(t)\right\}$, which contains any rectangle of the form $[\delta, T-\delta] \times\left[x_{0}-\xi, x_{0}+\xi\right]$ with $\xi<(3 / 4) \varepsilon$. Moreover, each of these rectangles can be fit into the interior of a smooth closed subset of $\Lambda_{\varepsilon, x_{0}}$ (see Figure 3 ).

Now there are cases (if $[a, b]$ is too long and $\varepsilon$-and consequently $\xi$-too small), where none of the rectangles $[\delta, T-\delta] \times\left[x_{0}-\xi, x_{0}+\xi\right]$ satisfies the geometric control condition (GCC). Thus we cannot apply Proposition 2.1 with controls supported in 


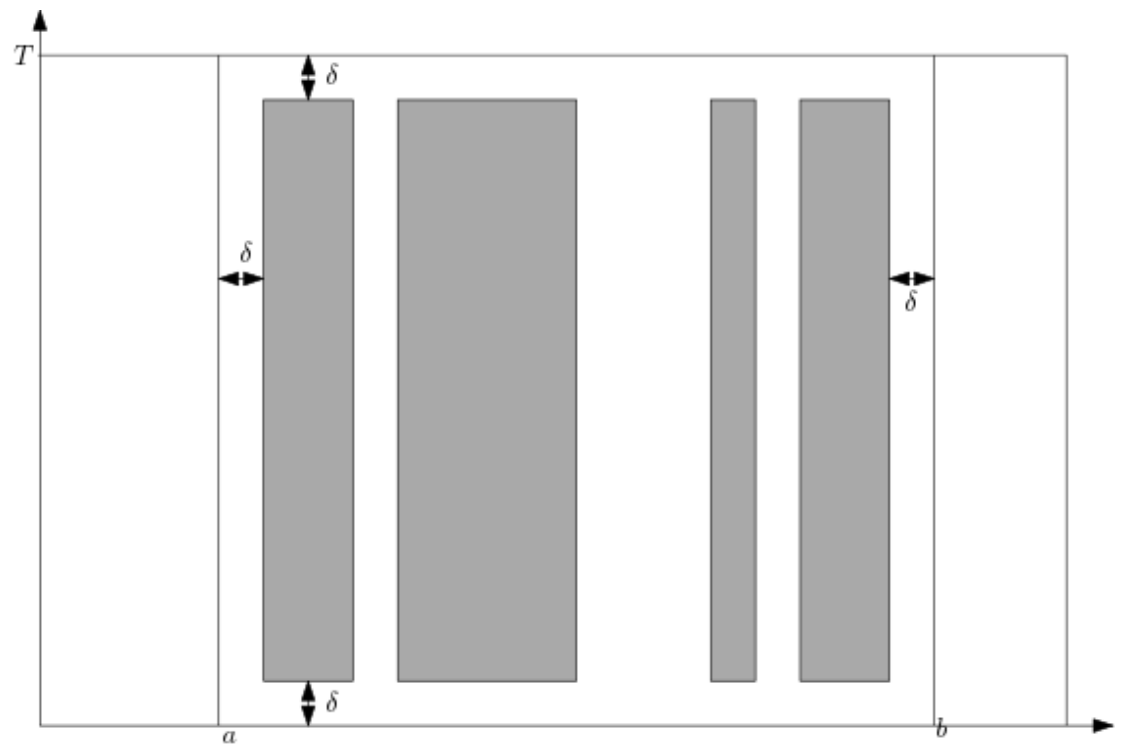

FIG. 4. An example of a $\delta$-covering set.

some $[\delta, T-\delta] \times\left[x_{0}-\xi, x_{0}+\xi\right]$, as time condition (3.52) does not hold in these cases. So we need to build a return trajectory $(\bar{u}, \bar{v}, \bar{h})$ such that $\bar{u} \neq 0$ on a smooth closed set $\mathcal{Q}$ containing a set $\mathcal{Q}_{\delta}$ that satisfies the GCC.

Now there is a simple type of set that would fit our needs for $\mathcal{Q}_{\delta}$ : in section 2 we worked in $[\delta, T-\delta] \times[a+\delta, b+\delta]$, but we do not need the whole rectangle in general for the GCC to be satisfied. We can in fact work with a number of much smaller rectangles, as long as they are close enough to each other (see Figure 4).

Definition 3.1. Let $0<\delta<\min ((b-a) / 4, T / 2)$, such that $(2.8)$ is satisfied. A $\delta$-covering set of $[0, T] \times[a, b]$ for system (1.1) is a union of rectangles of the form $\left\{[\delta, T-\delta] \times\left[a_{i}, b_{i}\right], 1 \leq i \leq N\right\}$ for some $N \geq 1$, such that

$$
\begin{gathered}
a_{1}=a+\delta, \\
b_{N}=b-\delta, \\
0<\left(a_{i+1}-b_{i}\right) \max \left(\frac{1}{\left|\nu_{1}\right|}, \frac{1}{\left|\nu_{2}\right|}\right)<T-2 \delta, \quad 1 \leq i \leq N-1 .
\end{gathered}
$$

Now the idea is to add the elementary trajectories obtained by the preliminary construction on disjoint supports centered in $x_{i} \in[a+\delta+\varepsilon, b-\delta-\varepsilon]$, that are close enough, and with a small enough $\varepsilon$ so that the rectangles $[\delta, T-\delta] \times\left[x_{0}-\varepsilon / 2, x_{0}+\varepsilon / 2\right]$ form a $\delta$-covering set (see Figure 5$)$. Take $\varepsilon_{0} \leq(b-a-2 \delta) / 2$ small enough for the preliminary construction to work, and such that $\varepsilon_{0} \max \left(1 / \nu_{1}, 1 / \nu_{2}\right)<T-2 \delta$. We then define the following sequence: take $N \in \mathbb{N}$ large enough so that

$$
\varepsilon:=\frac{b-a-2 \delta}{2 N-1} \leq \varepsilon_{0}
$$

and define, for $1 \leq i \leq N$,

$$
x_{i}:=a+\delta+\left(2 i-\frac{3}{2}\right) \varepsilon,
$$




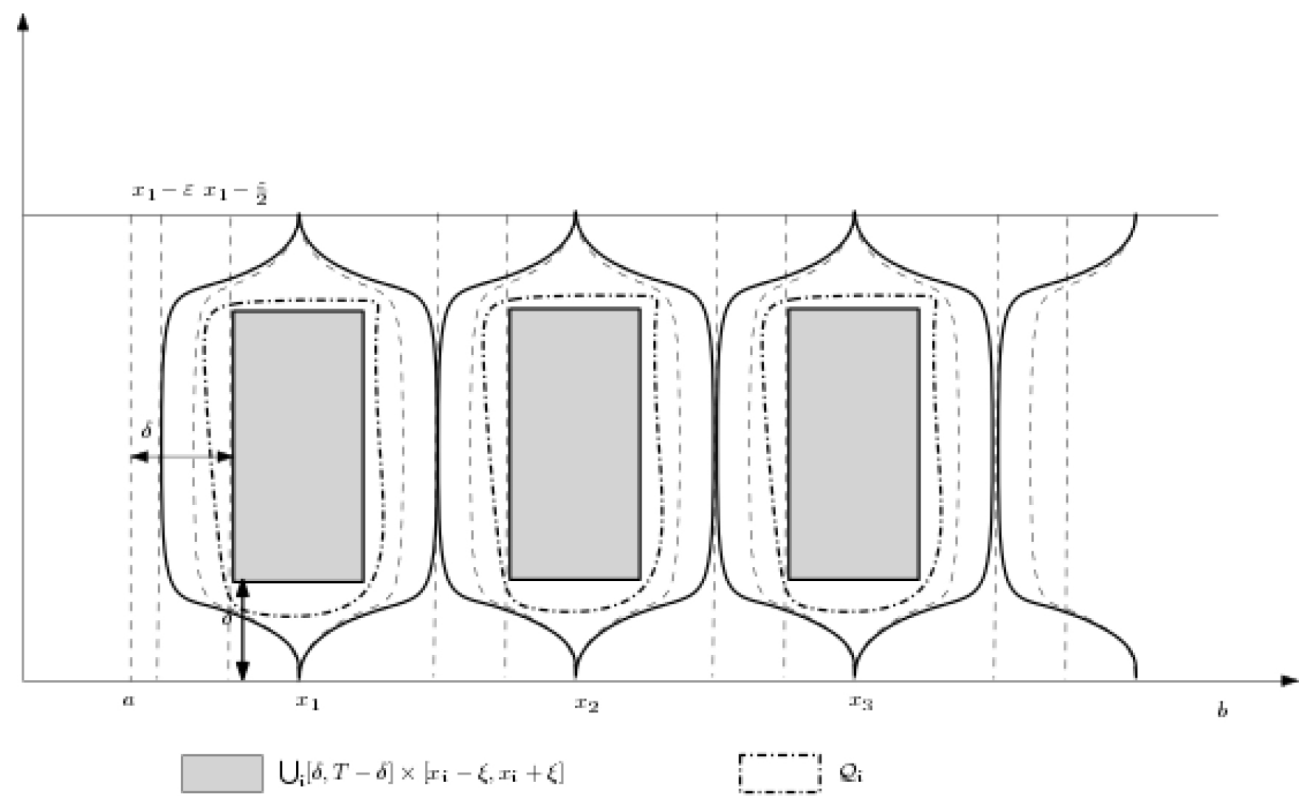

FIG. 5. Putting elementary trajectories side by side. The rectangles form a covering set.

and $\left(\bar{u}_{i}, \bar{v}_{i}, \bar{h}_{i}\right)$ the trajectory obtained by the preliminary construction corresponding to the chosen $\varepsilon$, centered in $x_{i}$. Let $\mathcal{Q}_{i}$ be a smooth closed subset of $\Lambda_{\varepsilon, x_{i}}$ containing $[\delta, T-\delta] \times\left[x_{i}-\varepsilon / 2, x_{i}+\varepsilon / 2\right]$ in its interior. Then,

$$
\mathcal{Q}_{\delta}:=\bigcup_{i=1}^{N}[\delta, T-\delta] \times\left[x_{i}-\varepsilon / 2, x_{i}+\varepsilon / 2\right]
$$

is a $\delta$-covering set,

$$
\mathcal{Q}:=\left(\bigcup_{i} \mathcal{Q}_{i}\right)
$$

is a smooth closed set such that $\mathcal{Q}_{\delta} \subset \stackrel{\circ}{\mathcal{Q}}$, and we can define

$$
(\bar{u}, \bar{v}, \bar{h}):=\sum_{i=1}^{N}\left(\bar{u}_{i}, \bar{v}_{i}, \bar{h}_{i}\right),
$$

which is supported in $[0, T] \times[a, b]$ and satisfies (3.2).

3.3. Local controllability with two controls and Gromov inversion. We now have our return trajectory $(\bar{u}, \bar{v}, \bar{h})$. Now let $R>0$, and notice that for all $\kappa>0$, $\left(\kappa \bar{u}, \kappa^{3} \bar{v}, \kappa \bar{h}\right)$ is also a return trajectory, with the same support. Thus, we can now suppose without loss of generality that

$$
\|(\bar{u}, \bar{v}, \bar{h})\|_{\left(C^{11}\right)^{3}} \leq \frac{R}{2} .
$$

Let $u, v \in C^{k}([0, T] \times[0, L]), h_{1}, h_{2} \in C^{k-2}([0, T] \times[0, L])$. Let us consider the trajectory $(\bar{u}+u, \bar{v}+v)$, controlled by $\left(\bar{h}+h_{1}, h_{2}\right)$. Then, we get the following control system for $u$ and $v$ : 


$$
\left\{\begin{aligned}
\square_{\nu_{1}} u & =h_{1}, \\
\square_{\nu_{2}} v & =u^{3}+3 \bar{u} u^{2}+3 \bar{u}^{2} u+h_{2}, \\
u(\cdot, 0) & =0, \\
u(\cdot, L) & =0, \\
v(\cdot, 0) & =0, \\
v(\cdot, L) & =0 .
\end{aligned}\right.
$$

This is a coupled semilinear system with a source term and falls in the category of systems (2.7). The aim of this section is to prove the following proposition.

Proposition 3.2. Let $k \geq 2,0 \leq a<b \leq L, T>0$ such that

$$
T>2(L-b) \max \left(\frac{1}{\nu_{1}}, \frac{1}{\nu_{2}}\right), T>2 a \max \left(\frac{1}{\nu_{1}}, \frac{1}{\nu_{2}}\right) .
$$

For every $0<\delta<\min (T / 2,(b-a) / 2)$ satisfying $(2.8)$, for every $\delta$-covering set $\mathcal{Q}_{\delta}$ of $[0, T] \times[a, b]$, there exists $\eta>0$ and constants $C_{1}, C_{2}>0$ depending on $T, \delta, k$ such that, for initial and final values

$\left(\left(u_{0}, u_{1}\right),\left(v_{0}, v_{1}\right),\left(u_{0}^{f}, u_{1}^{f}\right),\left(v_{0}^{f}, v_{1}^{f}\right)\right) \in\left(B_{C^{k}([0, T] \times[0, L])}(0, \eta) \times B_{C^{k-1}([0, T] \times[0, L])}(0, \eta)\right)^{4}$

satisfying (1.8) at the order $k$, there exist controls $h_{1}, h_{2} \in C^{k-1}([0, T] \times[0, L])$ satisfying

$$
\begin{array}{ll}
\operatorname{supp} h_{i} \subset \mathcal{Q}_{\delta}, & i=1,2, \\
\left\|h_{i}\right\|_{C^{k-2}} \leq C_{1}\left\|\left(\left(u_{0}, u_{1}\right),\left(v_{0}, v_{1}\right),\left(u_{0}^{f}, u_{1}^{f}\right),\left(v_{0}^{f}, v_{1}^{f}\right)\right)\right\|_{\left(C^{k} \times C^{k-1}\right)^{4}}, & i=1,2,
\end{array}
$$

such that the corresponding solution of $(2.7)$ with initial values $\left(\left(u_{0}, u_{1}\right),\left(v_{0}, v_{1}\right)\right)$ satisfies

$$
\begin{gathered}
\begin{cases}u(T, \cdot)=u_{0}^{f}, & u_{t}(T, \cdot)=u_{1}^{f}, \\
v(T, \cdot)=v_{0}^{f}, & v_{t}(T, \cdot)=v_{1}^{f},\end{cases} \\
\|(u, v)\|_{\left(C^{k}\right)^{2}} \leq C_{2}\left\|\left(\left(u_{0}, u_{1}\right),\left(v_{0}, v_{1}\right),\left(u_{0}^{f}, u_{1}^{f}\right),\left(v_{0}^{f}, v_{1}^{f}\right)\right)\right\|_{\left(C^{k} \times C^{k-1}\right)^{4}} .
\end{gathered}
$$

Remark 3.3. It is clear, by Definition 3.1, that for any $0<\delta<\min ((b-a) / 4, T / 2)$ such that (2.8) is satisfied, $[\delta, T-\delta] \times[a+\delta, b-\delta]$ is a $\delta$-covering set of $[0, T] \times[a, b]$. Thus Proposition 3.2 implies Proposition 2.1.

To prove this proposition, we use the following propositions, which are particular cases of more general quasi-linear results proved in [LR03] (see also [Li10, Chapter 5, sections 5.3 and 5.4]).

Proposition 3.3 (two-sided control). Let $k \geq 2, L>0, T>0, F \in C^{\infty}\left(\mathbb{R}^{2}, \mathbb{R}^{2}\right)$, $\nu_{1}, \nu_{2}>0$. If

$$
T>L \max \left(\frac{1}{\nu_{1}}, \frac{1}{\nu_{2}}\right),
$$

then there exists $\eta>0$ and a constant $C>0$ depending on $T, k$ such that for any initial and final values

$$
\left(U_{0}, U_{1}, U_{0}^{f}, U_{1}^{f}\right) \in B_{\left(C^{k}([0, L])^{2} \times C^{k-1}([0, L])^{2}\right)^{2}}(0, \eta)
$$


there exist controls $H_{1}$ and $H_{2} \in C^{k}\left([0, T], \mathbb{R}^{2}\right)$ satisfying compatibility conditions

$$
\left\{\begin{array}{rl}
P_{n, i}^{f_{i}}\left(J_{x}^{n}\left(U_{0}\right)(0), J_{x}^{n-1}\left(U_{1}\right)(0),(0, \ldots, 0)\right) & =\partial_{t}^{n} H_{1 i}(0), \\
P_{n, i}^{f_{i}}\left(J_{x}^{n}\left(U_{0}\right)(L), J_{x}^{n-1}\left(U_{1}\right)(L),(0, \ldots, 0)\right) & =\partial_{t}^{n} H_{2 i}(0), \\
P_{n, i}^{f_{i}}\left(J_{x}^{n}\left(U_{0}^{f}\right)(0), J_{x}^{n-1}\left(U_{1}^{f}\right)(0),(0, \ldots, 0)\right) & =\partial_{t}^{n} H_{1 i}(T), \\
P_{n, i}^{f_{i}}\left(J_{x}^{n}\left(U_{0}^{f}\right)(L), J_{x}^{n-1}\left(U_{1}^{f}\right)(L),(0, \ldots, 0)\right) & =\partial_{t}^{n} H_{2 i}(T),
\end{array} \forall n \leq k, i=1,2,\right.
$$

such that the solution to the vector system

$$
\left\{\begin{aligned}
\partial_{t t} U-\left(\begin{array}{cc}
\nu_{1}^{2} & 0 \\
0 & \nu_{2}^{2}
\end{array}\right) \partial_{x x} U & =F(U), x \in(0, L), \\
U(t, 0) & =H_{1} \\
U(t, L) & =H_{2} \\
U(0) & =U_{0} \\
U_{t}(0) & =U_{1}
\end{aligned}\right.
$$

satisfies

$$
\begin{gathered}
\left\{\begin{array}{c}
U(T)=U_{0}^{f}, \\
U_{t}(T)=U_{1}^{f},
\end{array}\right. \\
\|U\|_{C^{k}} \leq C\left\|\left(U_{0}, U_{1}\right),\left(U_{0}^{f}, U_{1}^{f}\right)\right\|_{\left(C^{k} \times C^{k-1}\right)^{2} .}
\end{gathered}
$$

Proposition 3.4 (one-sided control). Let $k \geq 2, L>0, T>0, F \in C^{\infty}\left(\mathbb{R}^{2}, \mathbb{R}^{2}\right)$, $\nu_{1}, \nu_{2}>0$. If

$$
T>2 L \max \left(\frac{1}{\nu_{1}}, \frac{1}{\nu_{2}}\right),
$$

then there exists $\eta>0$ and a constant $C>0$ depending on $T, k$ such that for any initial and final values

$$
\left(U_{0}, U_{1}, U_{0}^{f}, U_{1}^{f}\right) \in B_{\left(C^{k}([0, L])^{2} \times C^{k-1}([0, L])^{2}\right)^{2}}(0, \eta)
$$

there exists a control $H \in C^{k}\left([0, T], \mathbb{R}^{2}\right)$ satisfying compatibility conditions (3.43)

$$
\left\{\begin{array}{c}
P_{n, i}^{f_{i}}\left(J_{x}^{n}\left(U_{0}\right)(0), J_{x}^{n-1}\left(U_{1}\right)(0),(0, \ldots, 0)\right)=\partial_{t}^{n} H_{i}(0) \quad(\text { resp. }, 0), \\
P_{n, i}^{f_{i}}\left(J_{x}^{n}\left(U_{0}\right)(L), J_{x}^{n-1}\left(U_{1}\right)(L),(0, \ldots, 0)\right)=0 \quad\left(\text { resp., } \partial_{t}^{n} H_{i}(0)\right), \\
P_{n, i}^{f_{i}}\left(J_{x}^{n}\left(U_{0}^{f}\right)(0), J_{x}^{n-1}\left(U_{1}^{f}\right)(0),(0, \ldots, 0)\right)=\partial_{t}^{n} H_{i}(T) \quad(\text { resp., } 0), \\
P_{n, i}^{f_{i}}\left(J_{x}^{n}\left(U_{0}^{f}\right)(L), J_{x}^{n-1}\left(U_{1}^{f}\right)(L),(0, \ldots, 0)\right)=0 \quad\left(\text { resp., } \partial_{t}^{n} H_{i}(T)\right),
\end{array} \forall n \leq k, i=1,2,\right.
$$

such that the solution to the vector system

$$
\left\{\begin{aligned}
\partial_{t t} U-\left(\begin{array}{cc}
\nu_{1}^{2} & 0 \\
0 & \nu_{2}^{2}
\end{array}\right) \partial_{x x} U & =F(U), x \in(0, L), \\
U(t, L) & =0(\text { resp. }, U(t, L)=H(t)), \\
U(t, 0) & =H,(\text { resp. }, U(t, 0)=0), \\
U(0) & =U_{0}, \\
U_{t}(0) & =U_{1},
\end{aligned}\right.
$$


satisfies

$$
\begin{gathered}
\left\{\begin{array}{c}
U(T)=U_{0}^{f}, \\
U_{t}(T)=U_{1}^{f},
\end{array}\right. \\
\|U\|_{C^{k}} \leq C\left\|\left(U_{0}, U_{1}\right),\left(U_{0}^{f}, U_{1}^{f}\right)\right\|_{\left(C^{k} \times C^{k-1}\right)^{2} .}
\end{gathered}
$$

Proof of Proposition 3.2. Let us note

$$
\mathcal{Q}_{\delta}=\bigcup_{1 \leq i \leq N}[\delta, T-\delta] \times\left[a_{i}, b_{i}\right]
$$

for some $N \geq 1$. For every $1 \leq i \leq N-1$, let $0<\delta_{i}<\min \left(\left(b_{i+1}-a_{i+1}\right) / 2,\left(b_{i}-a_{i}\right) / 2\right)$ such that

$$
T-2 \delta_{i}>\left(a_{i+1}-b_{i}+4 \delta_{i}\right) \max \left(\frac{1}{\nu_{1}}, \frac{1}{\nu_{2}}\right) .
$$

Thanks to Propositions 3.3 and 3.4, Definition 3.1, and conditions (2.8) and (3.46), there exists $\eta>0$ such that for initial and final values

$$
\left(\left(u_{0}, u_{1}\right),\left(v_{0}, v_{1}\right),\left(u_{0}^{f}, u_{1}^{f}\right),\left(v_{0}^{f}, v_{1}^{f}\right)\right) \in\left(B_{C^{k}([0, L])}(0, \eta) \times B_{C^{k-1}([0, L])}(0, \eta)\right)^{4}
$$

satisfying (1.8),

- there exist boundary controls $u_{1}^{(i)}, u_{2}^{(i)} \in C^{k}([0, T-2 \delta])$ at $b_{i}-\delta_{i}$ and $a_{i+1}+\delta_{i}$ that steer $(u, v)$ on $\left[b_{i}-\delta_{i}, a_{i+1}+\delta_{i}\right]$ from $\left(y_{0}, y_{1}\right)_{\mid\left[b_{i}-\delta_{i}, a_{i+1}+\delta_{i}\right]}$ to $\left(z_{0}, z_{1}\right)_{\mid\left[b_{i}-\delta_{i}, a_{i+1}+\delta_{i}\right]}$ (see Figure 6 );

- there exist two boundary controls $u_{1}, u_{2} \in C^{k}([0, T-2 \delta])$ at $a+2 \delta$ and $b-2 \delta$ that steer $(u, v)$ on $[0, a+2 \delta]$ from $\left(y_{0}, y_{1}\right)_{\mid[0, a+2 \delta]}$ to $\left(z_{0}, z_{1}\right)_{\mid[0, a+2 \delta]}$ and from $\left(y_{0}, y_{1}\right)_{\mid[b-2 \delta, L]}$ to $\left(z_{0}, z_{1}\right)_{\mid[b-2 \delta, L]}$ while satisfying the boundary conditions of the system at 0 and $L$ (see Figure 6 ).

We note $\delta_{0}=\delta_{N}:=\delta$, and $\left(u^{*}, v^{*}\right)$ the corresponding trajectory on $[0, a+2 \delta] \cup[b-$ $2 \delta, L] \cup \bigcup_{1 \leq i \leq N-1}\left[b_{i}-\delta_{i}, a_{i+1}+\delta_{i}\right]$. Then, (3.45) and (3.42) imply

$$
\left\|\left(u^{*}, v^{*}\right)\right\|_{C^{k}} \leq C\left\|\left(u_{0}, u_{1}\right),\left(v_{0}, v_{1}\right),\left(u_{0}^{f}, u_{1}^{f}\right),\left(v_{0}^{f}, v_{1}^{f}\right)\right\|_{\left(C^{k} \times C^{k-1}\right)^{4}}
$$

for some constant $C>0$.

On the other hand, for $\eta>0$ small enough, for initial and final conditions $\left(\left(u_{0}, u_{1}\right),\left(v_{0}, v_{1}\right),\left(u_{0}^{f}, u_{1}^{f}\right),\left(v_{0}^{f}, v_{1}^{f}\right)\right) \in\left(B_{C^{k}([0, T] \times[0, L])}(0, \eta) \times B_{C^{k-1}([0, T] \times[0, L])}(0, \eta)\right)^{4}$, for $1 \leq i \leq N$ the forward evolving solutions $\left(u_{f}^{(i)}, v_{f}^{(i)}\right)$ of the vector equations

$$
\left\{\begin{aligned}
\square_{\nu_{1}} u & =f_{1}(\bar{u}+u, \bar{v}+v)-f_{1}(\bar{u}, \bar{v}), \\
\square_{\nu_{2}} v & =f_{2}(\bar{u}+u, \bar{v}+v)-f_{2}(\bar{u}, \bar{v}), \\
(u, v)\left(t, a_{i}\right) & =(u, v)\left(t, b_{i}\right)=(0,0), \\
(u, v)(0, \cdot) & =\left(u_{0 \mid\left[a_{i}, b_{i}\right]}, v_{0 \mid\left[a_{i}, b_{i}\right]}\right), \\
(u, v)_{t}(0, \cdot) & =\left(u_{1 \mid\left[a_{i}, b_{i}\right]}, v_{1 \mid\left[a_{i}, b_{i}\right]}\right)
\end{aligned}\right.
$$




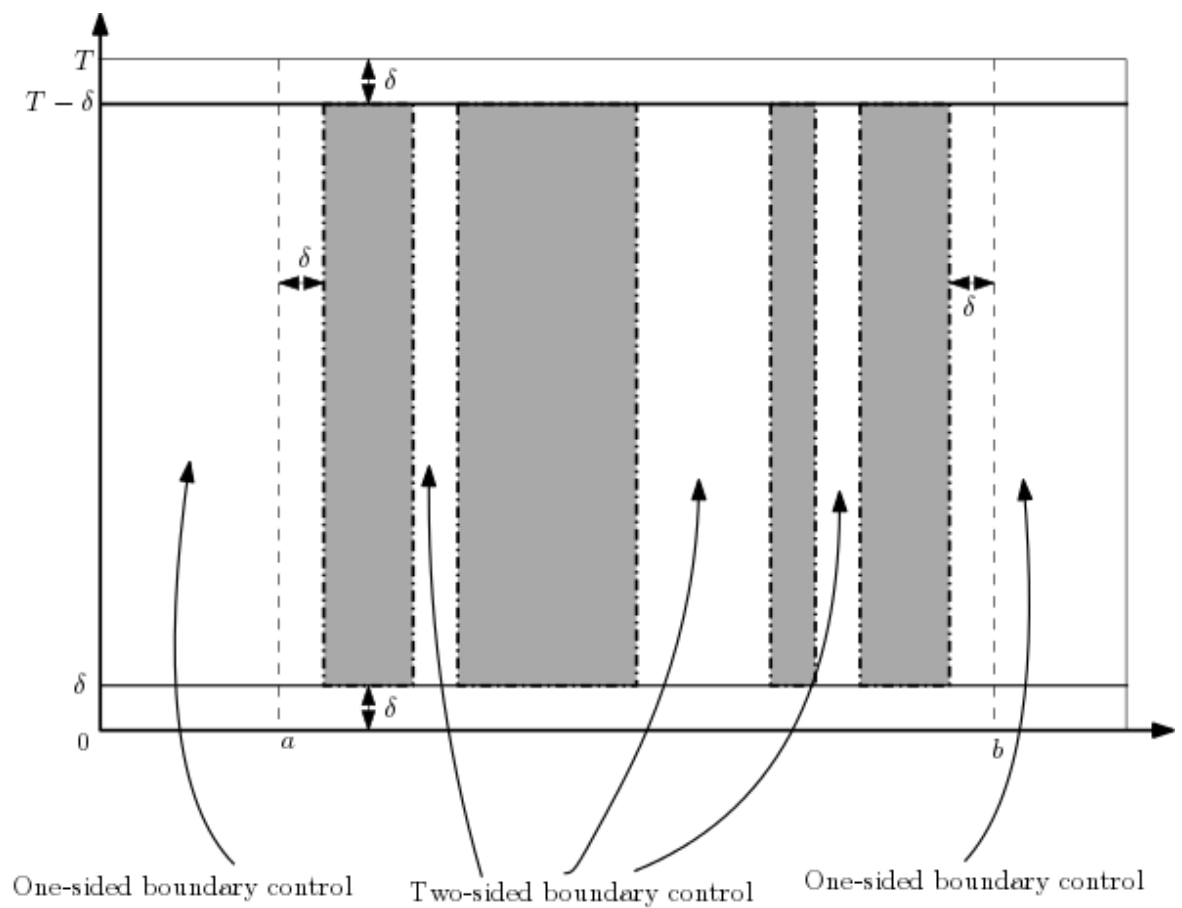

FIG. 6. Using boundary control results outside of the covering set.

are defined on $[0, T-2 \delta] \times\left[a_{i}, b_{i}\right]$. Let us also note $\left(u_{b}^{(i)}, v_{b}^{(i)}\right)$ the backward evolving solutions of the vector equations on $[0, T-2 \delta] \times\left[a_{i}, b_{i}\right]$

$$
\left\{\begin{aligned}
\square_{\nu_{1}} u & =f_{1}(\bar{u}+u, \bar{v}+v)-f_{1}(\bar{u}, \bar{v}), \\
\square_{\nu_{2}} v & =f_{2}(\bar{u}+u, \bar{v}+v)-f_{2}(\bar{u}, \bar{v}), \\
(u, v)\left(t, a_{i}\right) & =(u, v)\left(t, b_{i}\right)=(0,0), \\
(u, v)(T-2 \delta, \cdot) & =\left(u_{0 \mid\left[a_{i}, b_{i}\right]}^{f}, v_{0 \mid\left[a_{i}, b_{i}\right]}^{f}\right), \\
(u, v)_{t}(T-2 \delta, \cdot) & =\left(u_{1 \mid\left[a_{i}, b_{i}\right]}^{f}, v_{1 \mid\left[a_{i}, b_{i}\right]}^{f}\right) .
\end{aligned}\right.
$$

Then we define $(\tilde{u}, \tilde{v})$ by

$$
(\tilde{u}, \tilde{v})=\left(u_{f}^{(i)}, v_{f}^{(i)}\right) \phi+\left(u_{b}^{(i)}, v_{b}^{(i)}\right)(1-\phi), \text { on }\left[a_{i}, b_{i}\right], \forall i \leq N,
$$

where $\phi$ is a time cut-off function such that

$$
\phi(0)=1, \quad \phi(T-2 \delta)=0 .
$$

Note that, by well-posedness of the Cauchy problems, there exists $C^{\prime}>0$ such that the norm of $(\tilde{u}, \tilde{v})$ satisfies

$$
\|(\tilde{u}, \tilde{v})\|_{\left(C^{k}\right)^{2}} \leq C^{\prime}\left\|\left(u_{0}, u_{1}\right),\left(v_{0}, v_{1}\right),\left(u_{0}^{f}, u_{1}^{f}\right),\left(v_{0}^{f}, v_{1}^{f}\right)\right\|_{\left(C^{k} \times C^{k-1}\right)^{4}} .
$$

Finally, let us define $\left(u^{* *}, v^{* *}\right)$ by smoothly extending $\left(u^{*}, v^{*}\right)$ on $\bigcup_{1 \leq i \leq N}\left[a_{i}+\right.$ $\left.\delta_{i-1}, b_{i}-\delta_{i}\right]$ with

$$
\left\|\left(u^{* *}, v^{* *}\right)\right\|_{\left(C^{k}\right)^{2}} \leq C^{\prime \prime}\left\|\left(u^{*}, v^{*}\right)\right\|_{C^{k}}
$$


where $C^{\prime \prime}$ is a constant depending on the $a_{i}, b_{i}$. Then, we define $(u, v)$ by

$$
(u, v)=\xi\left(u^{* *}, v^{* *}\right)+(1-\xi)(\tilde{u}, \tilde{v}),
$$

where $\xi$ is a space cut-off function satisfying

$$
\begin{gathered}
\xi=1 \text { on }[0, a+\delta] \cup[b-\delta, L] \cup \bigcup_{1 \leq i \leq N-1}\left[b_{i}, a_{i+1}\right], \\
\xi=0 \text { on } \bigcup_{1 \leq i \leq N}\left[a_{i}+\delta_{i-1}, b_{i}-\delta_{i}\right] .
\end{gathered}
$$

Then, by construction, we have

$$
\left\{\begin{aligned}
u(0, \cdot) & =u_{0}, & v(0, \cdot) & =v_{0}, \\
u_{t}(0, \cdot) & =u_{1}, & v_{t}(0, \cdot) & =v_{1}, \\
u(T-2 \delta, \cdot) & =u_{0}^{f}, & v(T-2 \delta, \cdot) & =v_{0}^{f}, \\
u_{t}(T-2 \delta, \cdot) & =u_{1}^{f}, & v_{t}(T-2 \delta, \cdot) & =v_{1}^{f},
\end{aligned}\right.
$$

and

$$
\begin{aligned}
& \operatorname{supp}\left(\square_{\nu_{1}} u-f_{1}(\bar{u}+u, \bar{v}+v)-f_{1}(\bar{u}, \bar{v})\right) \subset \mathcal{Q}_{\delta}, \\
& \operatorname{supp}\left(\square_{\nu_{2}} v-f_{2}(\bar{u}+u, \bar{v}+v)-f_{2}(\bar{u}, \bar{v})\right) \subset \mathcal{Q}_{\delta} .
\end{aligned}
$$

Finally, (3.47), (3.48), and (3.49) imply that there exists a constant $C_{2}>0$ such that (3.39) holds, and, by continuity of the $f_{i}$, noting

$$
h_{i}:=\square_{\nu_{i}} u-f_{i}(\bar{u}+u, \bar{v}+v)-f_{i}(\bar{u}, \bar{v}), \quad i=1,2,
$$

there exists a constant $C_{1}>0$ such that (3.37) holds.

Now, we define

$$
\mathcal{A}=\left\{(u, v, h) \in\left(C^{2}(\mathcal{Q})\right)^{3} \mid \forall(t, x) \in \mathcal{Q}, u(t, x) \neq 0\right\},
$$

which is clearly nonempty, and

$$
\forall(u, v, h) \in C^{2}(\mathcal{Q})^{3}, \mathscr{D}(u, v, h)=\left(\square_{\nu_{1}} u-h, \square_{\nu_{2}} v-u^{3}\right) .
$$

Then, we have the following proposition, similar to Proposition 2.2.

Proposition 3.5. $\mathscr{D}$ admits an infinitesimal inversion of order 2 over $\mathcal{A}$.

Moreover, thanks to (3.33) and (3.2), we have the following.

Proposition 3.6.

$$
(\bar{u}, \bar{v}, \bar{h})_{\mid \mathcal{Q}} \in \mathcal{A} .
$$

Now, we can use Theorem 2.1: there exists $\eta>0$ such that for initial and final conditions

$$
\left(\left(u_{0}, u_{1}\right),\left(v_{0}, v_{1}\right),\left(u_{0}^{f}, u_{1}^{f}\right),\left(v_{0}^{f}, v_{1}^{f}\right)\right) \in\left(B_{C^{11}([0, L])}(0, \eta) \times B_{C^{10}([0, L])}(0, \eta)\right)^{4}
$$

the corresponding trajectories of system (3.35) with two controls $u^{*}, v^{*}, h_{1}, h_{2}$ are small enough in $\left(C^{11}\right)^{2} \times\left(C^{9}\right)^{2}$ norm so that $\mathscr{D}$ can be inverted locally around 
$\left(\bar{u}+u^{*}, \bar{v}+v^{*}, \bar{h}\right)$ and so that, by the continuity property, $(u, v, h):=\mathscr{D}_{\left(\bar{u}+u^{*}, \bar{v}+v^{*}, \bar{h}\right)}^{-1}$ $\left(\theta_{1}, \theta_{2}\right)$ satisfies

$$
\|(u-\bar{u}, v-\bar{v}, h-\bar{h})\|_{\left(C^{6}\right)^{3}} \leq \frac{R}{2} .
$$

Together with (3.34), this yields

$$
\|(u, v, h)\|_{\left(C^{6}\right)^{3}} \leq R .
$$

This proves the following local controllability result.

TheOREM 3.1. Let $R>0$, and $0 \leq a<b \leq L, T>0$ such that

$$
T>2(L-b) \max \left(\frac{1}{\left|\nu_{1}\right|}, \frac{1}{\left|\nu_{2}\right|}\right), T>2 a \max \left(\frac{1}{\left|\nu_{1}\right|}, \frac{1}{\left|\nu_{2}\right|}\right) .
$$

There exists $\eta>0$ such that for given initial and final conditions

$$
\left(\left(u_{0}, u_{1}\right),\left(v_{0}, v_{1}\right),\left(u_{0}^{f}, u_{1}^{f}\right),\left(v_{0}^{f}, v_{1}^{f}\right)\right) \in\left(B_{C^{11}([0, L])}(0, \eta) \times B_{C^{10}([0, L])}(0, \eta)\right)^{4}
$$

satisfying (1.8), there exists $h \in C^{6}([0, T] \times[0, L])$ satisfying

$$
\operatorname{supp} h \subset[0, T] \times[a, b]
$$

such that the corresponding solution $(u, v) \in C^{6}([0, T] \times[0, L])$ of $(1.2)$ with initial values $\left(\left(u_{0}, u_{1}\right),\left(v_{0}, v_{1}\right)\right)$ satisfies

$$
\left\{\begin{array}{l}
u(T, \cdot)=u_{0}^{f}, u_{t}(T, \cdot)=u_{1}^{f}, \\
v(T, \cdot)=v_{0}^{f}, v_{t}(T, \cdot)=v_{1}^{f}
\end{array}\right.
$$

and (3.51) holds.

Now let $\left(u_{0}, u_{1}, v_{0}, v_{1}, u_{0}^{f}, u_{1}^{f}, v_{0}^{f}, v_{1}^{f}\right) \in\left(C^{11}([0, L]) \times C^{10}([0, L])\right)^{4}$ such that (1.8) is satisfied. Let us note

$$
M:=\left\|\left(u_{0}, u_{1}, u_{0}^{f}, u_{1}^{f}\right)\right\|_{\left(C^{11} \times C^{10}\right)^{2}}+\left\|\left(v_{0}, v_{1}, v_{0}^{f}, v_{1}^{f}\right)\right\|_{\left(C^{11} \times C^{10}\right)^{2}}^{\frac{1}{3}},
$$

and $\alpha:=\frac{\eta}{2 M}$. Then,

$$
\begin{aligned}
& \left\|\alpha u_{0}\right\|_{C^{11}} \leq \eta, \quad\left\|\alpha u_{1}\right\|_{C^{10}} \leq \eta, \quad\left\|\alpha u_{0}^{f}\right\|_{C^{11}} \leq \eta, \quad\left\|\alpha u_{1}^{f}\right\|_{C^{10}} \leq \eta, \\
& \left\|\alpha^{3} v_{0}\right\|_{C^{11}} \leq \eta, \quad\left\|\alpha^{3} v_{1}\right\|_{C^{10}} \leq \eta, \quad\left\|\alpha^{3} v_{0}^{f}\right\|_{C^{11}} \leq \eta, \quad\left\|\alpha^{3} v_{1}^{f}\right\|_{C^{10}} \leq \eta,
\end{aligned}
$$

and these functions satisfy (1.8). We can now apply Theorem 3.1, and for any support and time $T>0$ compatible with that support, we get $(u, v, h)$ with initial and final conditions $\left(\alpha u_{0}, \alpha u_{1}, \alpha u_{0}^{f}, \alpha u_{1}^{f}, \alpha^{3} v_{0}, \alpha^{3} v_{1}, \alpha^{3} v_{0}^{f}, \alpha^{3} v_{1}^{f}\right)$ such that

$$
\left\{\begin{array}{l}
\square_{\nu_{1}} u=h, \\
\square_{\nu_{2}} v=u^{3}, \\
u_{\mid \partial \Omega}=0, \\
v_{\mid \partial \Omega}=0 .
\end{array}\right.
$$


Then we also have

$$
\left\{\begin{aligned}
\square_{\nu_{1}} \alpha^{-1} u & =\alpha^{-1} h, \\
\square_{\nu_{2}} \alpha^{-3} v & =\left(\alpha^{-1} u\right)^{3}, \\
\alpha^{-1} u(0) & =\alpha^{-1} u(L)=0, \\
\alpha^{-3} v(0) & =\alpha^{-3} v(L)=0 .
\end{aligned}\right.
$$

Thus, $\alpha^{-1} h$ steers $\left(u_{0}, u_{1}, v_{0}, v_{1}\right)$ to $\left(u_{0}^{f}, u_{1}^{f}, v_{0}^{f}, v_{1}^{f}\right)$ in $T$.

Finally, to get estimate (1.14), recall (3.51),

$$
\|h\|_{C^{6}} \leq R,
$$

hence, in terms of the original control system,

$$
\begin{aligned}
\left\|\alpha^{-1} h\right\|_{C^{6}} & \leq \alpha^{-1} R \\
& \leq \frac{2 R}{\eta}\left(\left\|\left(u_{0}, u_{1}, u_{0}^{f}, u_{1}^{f}\right)\right\|_{\left(C^{11} \times C^{10}\right)^{2}}+\left\|\left(v_{0}, v_{1}, v_{0}^{f}, v_{1}^{f}\right)\right\|_{\left(C^{11} \times C^{10}\right)^{2}}^{\frac{1}{3}}\right) .
\end{aligned}
$$

This proves Theorem 1.2.

3.4. A general criterion for internal controllability. Let us now give a general definition, which gives the main criterion our return trajectories must fulfill to apply our method.

Definition 3.2. A suitable return trajectory for time $T>0$ is a trajectory $(\bar{u}, \bar{v}, \bar{h}) \in C^{11}([0, T] \times[0, L])^{3}$ of system $(1.1)$ such that

$$
\begin{gathered}
\bar{u}(0, \cdot)=0, \bar{v}(0, \cdot)=0, \\
\bar{u}_{t}(0, \cdot)=0, \bar{v}_{t}(0, \cdot)=0, \\
\bar{u}(T, \cdot)=0, \bar{v}(T, \cdot)=0, \\
\bar{u}_{t}(T, \cdot)=0, \bar{v}_{t}(T, \cdot)=0, \\
\operatorname{supp}(\bar{u}, \bar{v}, \bar{h}) \subset[0, T] \times[a, b], \\
\mathscr{D}(\bar{u}, \bar{v}, \bar{h})=(0,0),
\end{gathered}
$$

and such that there exists $0<\delta<\min (T / 2,(b-a) / 2)$ satisfying $(2.8)$, a $\delta$-covering set $\mathcal{Q}_{\delta}$, a smooth closed set $\mathcal{Q}$ such that $\mathcal{Q}_{\delta} \subset \stackrel{\circ}{\mathcal{Q}}$ such that

$$
\forall(t, x) \in \mathcal{Q}, \bar{u}(t, x) \neq 0 .
$$

We can now give a general statement to sum up our work on system (1.2).

Proposition 3.7. Let $0 \leq a<b \leq L$, and $T>0$ such that (1.9) holds. Suppose condition (1.10) does not hold. If one can find a suitable return trajectory, then system (1.1) is locally controllable in time $T$ for $\left(C^{11} \times C^{10}\right)^{4}$ initial and final conditions, with $C^{6}$ trajectories, and with a $C^{6}$ control supported in $[0, T] \times[a, b]$.

\section{Further questions.}

4.1. Regularity. Our method requires somewhat specific regularities: $C^{11}\left(C^{10}\right.$ for the time derivative) for the initial and final data. As is often the case when using a Nash-Moser scheme, these regularities are probably not optimal. However, if we require, for example, $C^{k}$ regularity for the control, $k \geq 2$, the initial and final data have to be at least one notch smoother. Indeed, note

$$
w:=u_{t}-\nu_{1} u_{x}
$$


and consider the following computation, where one requires the control and the trajectories to be $C^{k}$ :

$$
\begin{aligned}
\frac{d}{d t} w\left(t, x-\nu_{1} t\right) & =w_{t}-\nu_{1} w_{x} \\
& =w_{t}+\nu_{1} w_{x}-2 \nu_{1} w_{x} \\
& =h\left(t, x-\nu_{1} t\right)-2 \nu_{1}\left(u_{t x}-\nu_{1} u_{x x}\right) \\
& =h\left(t, x-\nu_{1} t\right)-2 \nu_{1} \frac{d}{d t} u_{x}\left(t, x-\nu_{1} t\right) ;
\end{aligned}
$$

hence, for a fixed $t>0$ and for characteristics going from $\{0\} \times[0, L]$ to $\{t\} \times[0, L]$,

$$
\begin{aligned}
\int_{0}^{t} h\left(s, x-\nu_{1} s\right) d s= & u_{t}\left(t, x-\nu_{1} t\right)-u_{1}(x)-\nu_{1} u_{x}\left(t, x-\nu_{1} t\right)+\nu_{1} u_{0}^{\prime}(x) \\
& -2 \nu_{1}\left(u_{x}\left(t, x-\nu_{1} t\right)-u_{0}^{\prime}(x)\right) .
\end{aligned}
$$

Now the left-hand side of (4.1) is a $C^{k}$ function of $x$, so we need to upgrade the regularity of $u_{0}$ to $C^{k+1}$ and that of $u_{1}, x \mapsto u_{x}(t, x)$, and $x \mapsto u_{t}(t, x)$ to $C^{k}$. This shows a partial derivative loss (in the space dimension) between the trajectory and the control and, taking $t=T$, a derivative loss between the initial and final data for $u$ and the control.

For linear cascade systems with smooth coefficients, the same procedure can be repeated on the second equation to establish similar losses of derivatives, showing that because $u$ has increased spatial regularity, the initial and final data for $v$ have to be $C^{k+2} \times C^{k+1}$. Note that with two controls, this would not be the case, as each control "absorbs" the loss of derivatives in each equation.

Furthermore, it would be interesting to consider other iteration schemes such as the one presented in [Cor07, section 4.2.1], where one considers the following linear system:

$$
\left\{\begin{array}{l}
\square u=f_{1}(0,0)+g_{1}^{v}(a, b) v+g_{1}^{u}(a, 0) u+h, \\
\square v=f_{2}(0,0)+g_{2}^{v}(0, b) v+g_{2}^{u}(a, b) u,
\end{array}\right.
$$

where, for $i \in\{1,2\}$,

$$
\begin{array}{r}
g_{i}^{u}(u, v)=\left\{\begin{array}{c}
\frac{f_{i}(u, v)-f_{i}(0, v)}{u} \text { for } u \neq 0, \\
\partial_{u} f_{i}(0, v) \text { for } u=0,
\end{array}\right. \\
g_{i}^{v}(u, v)=\left\{\begin{array}{c}
\frac{f_{i}(u, v)-f_{i}(u, 0)}{v} \text { for } v \neq 0, \\
\partial_{v} f_{i}(u, 0) \text { for } v=0 .
\end{array}\right.
\end{array}
$$

Then, by superposition one can restrict to the study of

$$
\left\{\begin{array}{l}
\square u=h, \\
\square v=g_{2}^{u}(a, b) u .
\end{array}\right.
$$

But ultimately, this only shifts the problem of the $C^{k}$ regularity gap between data and control, although we now have a linear system instead of a semilinear one. 
On the other hand, there are some situations where the Nash-Moser theorem eventually proves unnecessary. The best-known example for this improvement is geometric: in [G89] and [G91], it was proved that the problem of isometric embedding could be solved using a classical iteration scheme, instead of a Nash-Moser one. More recently, in control theory, controllability results for the Schrödinger equation were similarly improved. As mentioned in the introduction, controllability results for the 1-D Schrödinger equation were first proved using a Nash-Moser implicit function theorem [Bea05], [BC06], because of an a priori loss of derivatives. Beauchard and Laurent later discovered that, because of a regularizing effect, there was actually no loss of derivatives, and they proved more general results on the bilinear control of the linear and nonlinear 1-D Schrödinger equation in [BL10], using a classical inversion theorem on optimal function spaces. It would be interesting to know if a similar do-over is possible for our result, keeping in mind that the argument we gave above excludes the possibility of a regularizing effect that would cancel all loss of derivatives. We could hope for a result with a $C^{2}$ control and $\left(C^{4} \times C^{3}\right)^{2}$ initial and final conditions, for example.

This would also be interesting in terms of numerical analysis, to compute approximate controls and trajectories for the considered system. Indeed, the proof of Gromov's inversion theorem relies on a Nash-Moser type iterative scheme, which could be computed. However, such an iterative scheme would be very heavy to implement, because of the regularization at each step. A classical inversion theorem, relying on a Newton scheme, would be lighter to implement.

Finally, it would be interesting to investigate a $H^{k}$ version of this result, using other versions of the Nash-Moser implicit function theorem.

4.2. Other systems with an uncontrollable linearized system. Our scheme of proof also allows us to prove a controllability result for systems of the form

$$
\left\{\begin{array}{l}
\square_{\nu_{1}} u=G(u, v)+h, G \in C^{\infty}\left(\mathbb{R}^{2}\right), \\
\square_{\nu_{2}} v=u^{3}, \\
u_{\mid \partial \Omega}=0, \\
v_{\mid \partial \Omega}=0 .
\end{array}\right.
$$

Indeed, this simply adds a term in the definition of $\bar{h}$ when we build our return trajectory. However, $\bar{h}$ is no longer supported in $[0, T] \times[a, b]$. The other steps remain unchanged, as the additional $G$ term does not prevent the differential operator $\mathscr{D}$ from being algebraically solvable. So we get a local internal controllability result with the same time conditions, but no condition on the support of the control. Finally, if $G$ is homogeneous of degree 1, we can use the scaling argument to deduce a global result.

In addition to adding a coupling term to the first equation, we can also change the power of the coupling term in the second equation. There are two cases:

1. Even powers. As such, our method cannot work for even powers: indeed, $u^{2 k}$ has nonnegative values. In particular, by the same convexity argument as in Remark 3.2, solutions to the stationary problem cannot vanish smoothly in 1 . So the perturbative approach would allow us to build smooth return trajectories only if $u$ (and thus $h$ ) is spatially supported in all of $[0, L]$.

Another way of answering this question would be to switch to complex values, as is done in the appendix of [CGR10] for the quadratic case.

2. Odd powers. Thanks to Proposition 3.7, we know that the part that requires the most work is the construction of return trajectories: say the power of the 
coupling is $2 k+1, k \in \mathbb{N}^{*}$; in order to control all the derivatives of $v$ up to $2 k+1$, we would have to look for $v$ in the form

$$
\sum_{i=0}^{2 k+1} f_{i}(t) g_{i}\left(\frac{\left|x-\frac{b+a}{2}\right|}{\lambda}\right) \text {. }
$$

This would call for ever longer computations, and for now there is no indication that there might or might not be new difficulties with these additional terms.

4.3. Boundary controllability. In this article we have explored a method to prove internal controllability with one control. However, to our knowledge there is no result for boundary controllability with one control for semilinear systems such as (1.1). Although boundary controllability is relatively easy to establish for simple equations, or when there are the same numbers of controls and equations, we cannot use results on the inversion of differential operators to reduce the number of controls.

Acknowledgments. I would like to thank Jean-Michel Coron, who suggested that I work on this problem, for his continuous support and valuable remarks. I would also like to thank Shengquan Xiang, Amaury Hayat, Pierre Lissy, and Frédéric Marbach for our discussions on this problem. Finally, I would like to express my gratitude toward ETH-FIM for their generous support and their hospitality.

\section{REFERENCES}

[AB13] F. Alabau-Boussouira, A hierarchic multi-level energy method for the control of bidiagonal and mixed n-coupled cascade systems of PDE's by a reduced number of controls, Adv. Differential Equations, 18 (2013), pp. 1005-1072.

[AB14] F. AlABAU-Boussouira, Insensitizing exact controls for the scalar wave equation and exact controllability of 2-coupled cascade systems of PDE's by a single control, Math. Control Signals Systems, 26 (2014), pp. 1-46.

[ABCO17] F. Alabau-Boussoura, J.-M. Coron, And G. Olive, Internal controllability of first order quasi-linear hyperbolic systems with a reduced number of controls, SIAM J. Control Optim., 55 (2017), pp. 300-323.

[ABL13] F. Alabau-Boussouira and M. LÉautaud, Indirect controllability of locally coupled wave-type systems and applications, J. Math. Pures Appl. (9), 99 (2013), pp. 544-576.

[BC06] K. BeAuChard And J.-M. Coron, Controllability of a quantum particle in a moving potential well, J. Funct. Anal., 232 (2006), pp. 328-389.

[Bea05] K. BeAuchard, Local controllability of a 1-D Schrödinger equation, J. Math. Pures Appl. (9), 84 (2005), pp. 851-956.

[BL10] K. BeAUChaRd AND C. LAURENT, Local controllability of $1 D$ linear and nonlinear Schrödinger equations with bilinear control, J. Math. Pures Appl. (9), 94 (2010), pp. 520-554.

[BLR92] C. Bardos, G. Lebeau, and J. Rauch, Sharp sufficient conditions for the observation, control, and stabilization of waves from the boundary, SIAM J. Control Optim., 30 (1992), pp. 1024-1065.

[CG17] J.-M. Coron AND J.-P. GullLeron, Control of three heat equations coupled with two cubic nonlinearities, SIAM J. Control Optim., 55 (2017), pp. 989-1019.

[CGR10] J.-M. CORON, S. Guerrero, AND L. Rosier, Null controllability of a parabolic system with a cubic coupling term, SIAM J. Control Optim., 48 (2010), pp. 5629-5653.

[Che76] W. C. Chewning, Controllability of the nonlinear wave equation in several space variables, SIAM J. Control Optim., 14 (1976), pp. 19-25.

[CKL99] P. Cannarsa, V. Komornik, and P. Loreti, Controllability of semilinear wave equations with infinitely iterated logarithms, Control Cybernet., 28 (1999), pp. 449-461. 
[CKL02] P. Cannarsa, V. Komornik, and P. Loreti, One-sided and internal controllability of semilinear wave equations with infinitely iterated logarithms, Discrete Contin. Dyn. Syst., 8 (2002), pp. 745-756.

[CL14] J.-M. CORON AND P. LISSY, Local null controllability of the three-dimensional NavierStokes system with a distributed control having two vanishing components, Invent. Math., 198 (2014), pp. 833-880.

[Cor92] J.-M. Conon, Global asymptotic stabilization for controllable systems without drift, Math. Control Signals Systems, 5 (1992), pp. 295-312.

[Cor93] J.-M. Coron, Contrôlabilité exacte frontière de l'équation d'Euler des fluides parfaits incompressibles bidimensionnels, C. R. Acad. Sci. Paris, 317 (1993), pp. 271-276.

[Cor96] J.-M. Conon, On the controllability of 2-D incompressible perfect fluids. J. Math. Pures Appl. (9), 75 (1996), pp. 155-188.

[Cor07] J.-M. Conon, Control and Nonlinearity, Math. Surveys Monogr. 136, AMS, Providence, RI, 2007.

[Fat75] H. O. FAtToRini, Local controllability of a nonlinear wave equation, Math. Systems Theory, 9 (1975), pp. 30-45.

[FYZ07] X. FU, J. YONG, AND X. ZHANG, Exact controllability for multidimensional semilinear hyperbolic equations, SIAM J. Control Optim., 46 (2007), pp. 1578-1614.

[G89] M. GüNTHER, On the perturbation problem associated to isometric embeddings of Riemannian manifolds, Ann. Global Anal. Geom., 7 (1989), pp. 69-77.

[G91] M. GüNTHER, Isometric embeddings of Riemannian manifolds, in Proceedings of the International Congress of Mathematicians, Kyoto, Mathematical Society of Japan, Tokyo, 1991, pp. 1137-1143.

[GBPGa05] M. GonzÁlez-Burgos and R. PÉrez-Garcí A, Controllability of some coupled parabolic systems by one control force, C. R. Math. Acad. Sci. Paris, 340 (2005), pp. $125-130$.

[Gro86] M. Gromov, Partial Differential Relations, Ergeb. Math. Grenzgeb. (3) 9, SpringerVerlag, Berlin, 1986.

[HI11] S. W. HANSEN AND O. IMANuvilov, Exact controllability of a multilayer Rao-Nakra plate with clamped boundary conditions, ESAIM Control Optim. Calc. Var., 17 (2011), pp. 1101-1132.

[Isi95] A. Isidori, Nonlinear Control Systems, Comm. Control Engrg. Ser., Springer-Verlag, Berlin, 3rd ed., 1995.

[KL00] V. Komornik And P. Loreti, Partial observability of coupled linear systems, Acta Math. Hungar., 86 (2000), pp. 49-74.

[Li10] T.-T. LI, Controllability and Observability for Quasilinear Hyperbolic Systems, AIMS Ser. Appl. Math. 3, American Institute of Mathematical Sciences, Springfield, MO, 2010.

[Lio88a] J.-L. Lions, Contrôlabilité exacte, perturbations et stabilisation de systèmes distribués. Tome 1, Recherches Math. Appl. 8, Masson, Paris, 1988.

[Lio88b] J.-L. Lions, Contrôlabilité exacte, perturbations et stabilisation de systèmes distribués. Tome 2, Recherches Math. Appl. 9, Masson, Paris, 1988.

[LR03] T.-T. LI AND B.-P. RAO, Exact boundary controllability for quasi-linear hyperbolic systems, SIAM J. Control Optim., 41 (2003), pp. 1748-1755.

[NvdS90] H. Nijmeijer AND A. van DeR Schaft, Nonlinear Dynamical Control Systems, Springer-Verlag, New York, 1990.

[Teb11] L. Tebou, Some results on the controllability of coupled semilinear wave equations: The desensitizing control case, SIAM J. Control Optim., 49 (2011), pp. 1221-1238.

[Zha00a] X. ZhANG, Explicit observability estimate for the wave equation with potential and its application, R. Soc. Lond. Proc. Ser. A Math. Phys. Eng. Sci., 456 (2000), pp. 1101-1115.

[Zha00b] X. ZHANG, Explicit observability inequalities for the wave equation with lower order terms by means of Carleman inequalities, SIAM J. Control Optim., 39 (2000), pp. 812-834.

[Zua91] E. ZuAZUA, Exact boundary controllability for the semilinear wave equation, in Nonlinear Partial Differential Equations and Their Applications, Collège de France Seminar, Vol. X (Paris, 1987-1988), Pitman Res. Notes Math. Ser. 220, Longman, Harlow, UK, 1991, pp. 357-391.

[Zua93] E. ZuAZuA, Exact controllability for semilinear wave equations in one space dimension, Ann. Inst. H. Poincaré Anal. Non Linéaire, 10 (1993), pp. 109-129. 